\title{
Performance Estimation and Fault Diagnostics for the Starter of Auxiliary Power Unit
}

\author{
by
}

Yu Zhang

A thesis submitted to the Faculty of Graduate and Postdoctoral Affairs in partial fulfillment of the requirements for the degree of

Master of Applied Science

in

Aerospace Engineering

Carleton University

Ottawa, Ontario

(C) 2018 submitted, Yu Zhang 


\section{Abstract}

An electric starter provides initial power to run the Auxiliary Power Unit (APU) during the starting process. As the starter degrades, its output power declines which, as a result, affects the APU starting performance, and eventually leads to the starting failure. Therefore, a reliable starter health condition monitoring system is necessary to estimate the starter's degradation, diagnose its fault, and prevent its malfunction. Some research works have been reported in the literature for starter performance estimation and fault diagnostics, however, these prior works typically employ statistical analysis and datadriven methods, which are not physically interpretable and in some cases, are not reliable. Accordingly, the objective of this thesis is to develop a physics-based diagnostic scheme for the starter performance degradation estimation to improve fault diagnostics reliability. In this study, a novel physics-based transient model associated with the APU starting process is developed to reveal the physical link between APU performance parameters and the starter degradation. This model involves both the starter and the APU and considers kinetic energy during the startup phase. The performance of this physics-based diagnostic model is compared with that of a neural-network-based data-driven model under both healthy and faulty conditions. To quantify the degradation severity of the starter, two specific health indicators are derived from the proposed physics-based model. They both possess explicit physical meaning and perform fault diagnostics at the system level and component level, respectively. To further improve the fault diagnostics reliability, an enhanced joint indicator is also proposed to aggregate two individual indicators to detect the starter failure in a two-dimensional feature space and thereby 
enabling multiple-feature diagnostics. Finally, the receiver operating characteristic (ROC) curves are utilized to evaluate the diagnostic performance of the proposed health indicators and determine the optimal fault threshold. The developed physics-based performance estimation and fault diagnostics scheme for the starter of APUs supports the condition-based maintenance (CBM) strategy, and possesses a great potential for diagnostic reliability enhancement and considerable operational cost savings. 


\section{Acknowledgements}

I would like to sincerely acknowledge my supervisor Professor Jie Liu for his encouragement and advice during my Master's program. Without his enthusiastic supervision and patient guidance, this work could not be completed.

I am grateful to Dr. Houman Hanachi for his instructive guidance and tremendous assistance and Dr. Chunsheng Yang for his data support and interpretations. Many thanks to my friends Jinwei Yang, Rui Zhao, Zelig Li, Xiaozhou Zhang, Kun Zhuang and Yu Ji for their great helps.

I thank my parents and parents-in-law for their encouragement and support. Above all, I am indebted to my beloved wife Yining Yang and our daughter Zhiyu Zhang for their understanding and endless love. 


\section{Table of Contents}

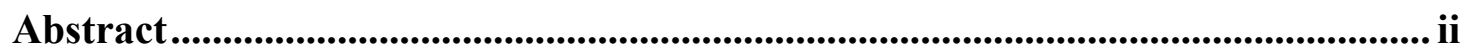

Acknowledgements...................................................................................................................... iv

Table of Contents ......................................................................................................................... v

List of Tables ...................................................................................................................... viii

List of Figures ........................................................................................................................... ix

List of Symbols ........................................................................................................................... xi

1 Chapter: Introduction .................................................................................................... 1

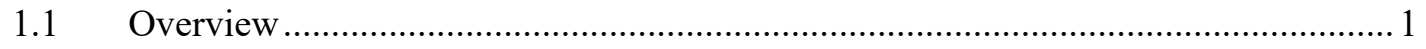

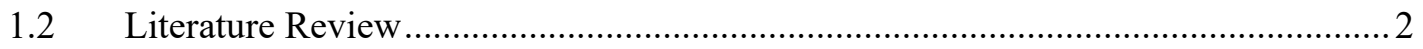

1.2.1 Physics-based Model for Diagnostics..................................................................

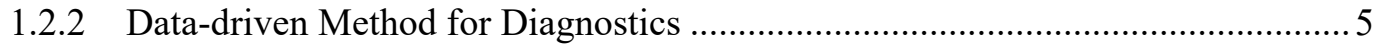

1.2.3 Previous Works on the Starter Fault Diagnostics ................................................

1.3 Thesis Objectives ...................................................................................

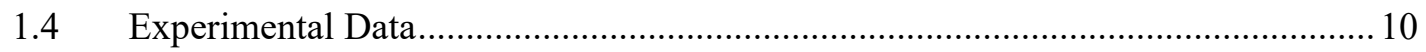

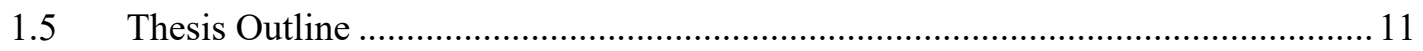

2 Chapter: Physics-based Model for APU Performance Monitoring ................. 12

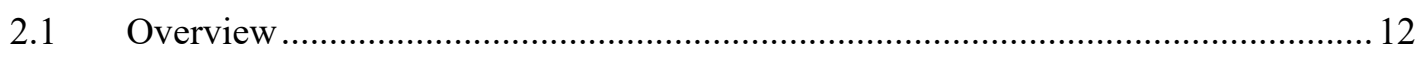

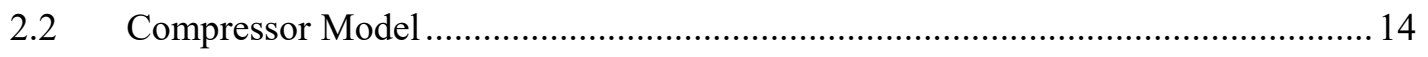

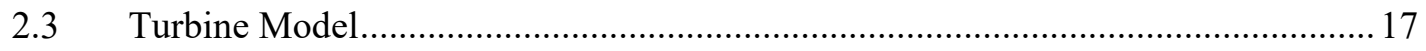

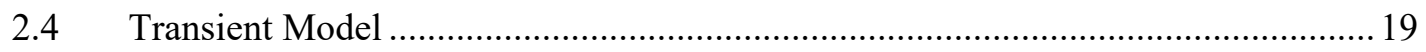

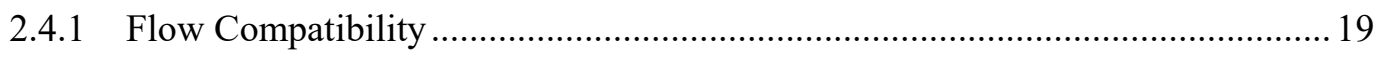

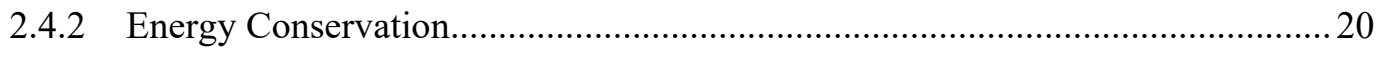

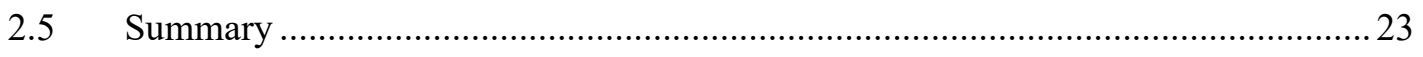


3 Chapter: Neural Network Model for APU Performance Monitoring............. 25

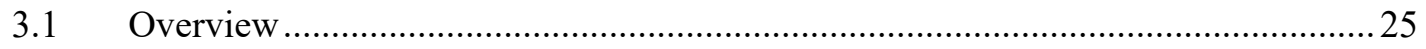

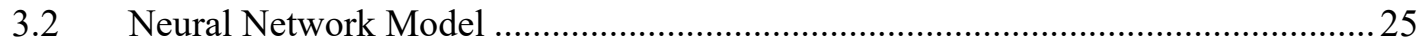

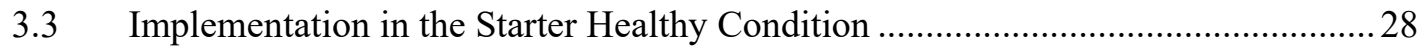

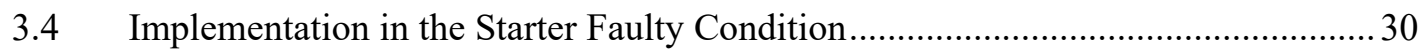

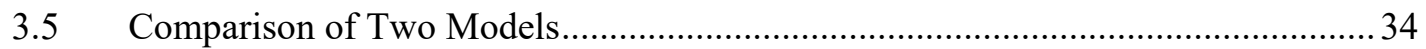

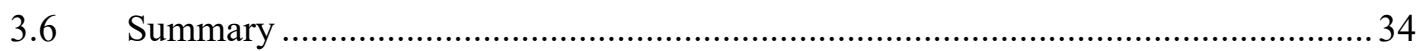

4 Chapter: Health Indicators for Starter Health Monitoring........................... 36

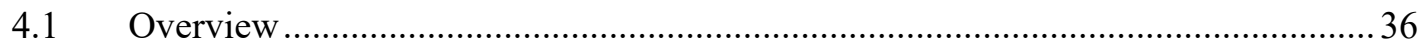

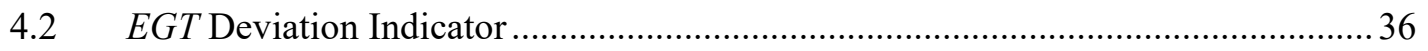

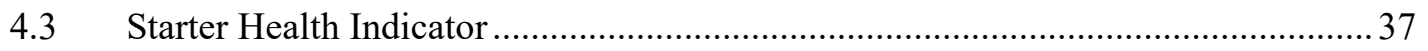

4.4 Implementation Results for EGT Deviation Indicator ...................................... 40

4.5 Implementation Results for Starter Health Indicator ........................................... 43

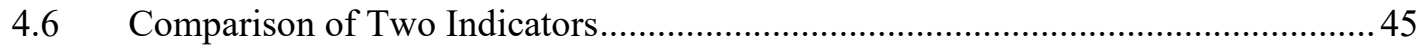

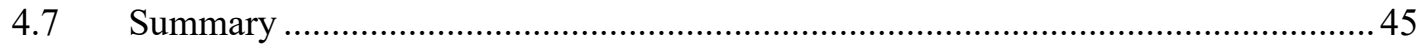

5 Chapter: Starter Fault Diagnostics …..................................................................... 47

$5.1 \quad$ Implementation of Individual Indicators for Diagnosis ...................................... 47

5.2 Implementation of An Enhanced Joint Indicator for Diagnosis .............................. 53

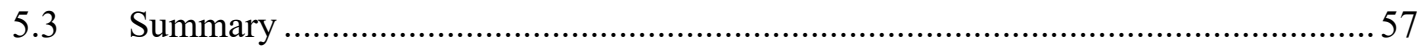

6 Chapter: Evaluation of Diagnostic Results for Indicators ............................. 59

6.1 Receiver Operating Characteristic Curves ............................................................ 59

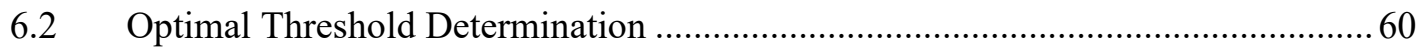

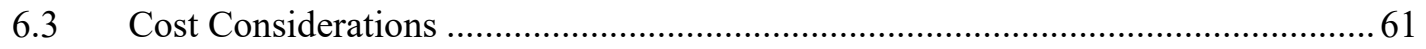

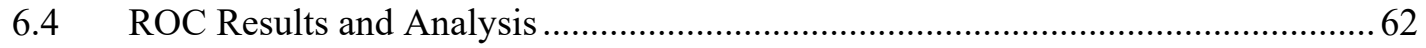

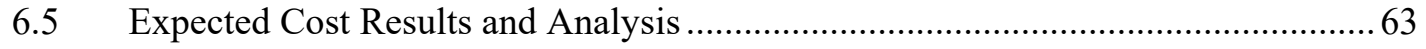

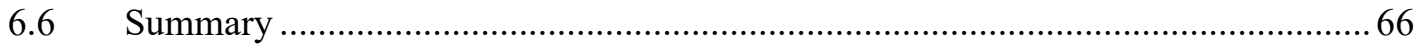


7 Chapter: Conclusions and Future work .....................................................6 67

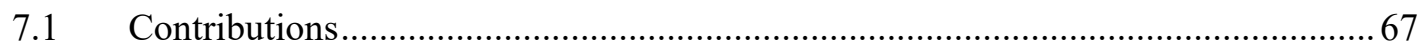

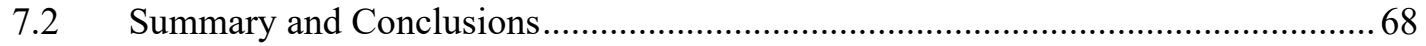

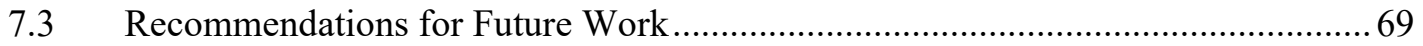

Bibliography or References.......................................................................... 70 


\section{List of Tables}

Table 1.1 Parameters of APU at the design point .............................................. 10

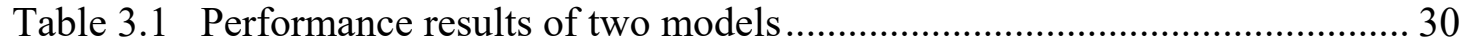

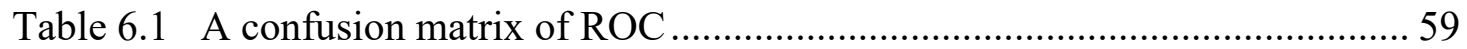

Table 6.2 Confusion matrices for the three indicators ........................................ 64 


\section{List of Figures}

Figure 2.1 Thermodynamic cycle of the APU................................................... 13

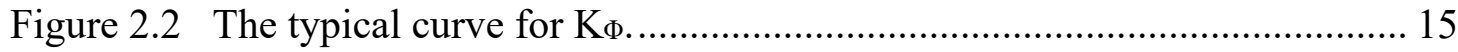

Figure 2.3 Critical pressure ratio in approximate turbine characteristics ................. 18

Figure 2.4 Procedures for implementing the transient model ............................... 23

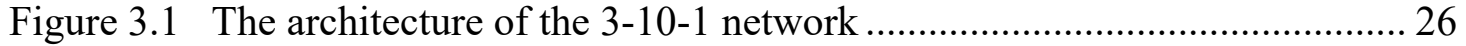

Figure 3.2 Training procedures of the neural network.......................................... 28

Figure 3.3 Implementation in healthy condition with physics-based model. ............ 29

Figure 3.4 Implementation in healthy condition with neural network model............ 29

Figure 3.5 Implementation in faulty condition with physics-based model............... 32

Figure 3.6 Implementation in faulty condition with neural network model............. 33

Figure 4.1 APU starter performance during the startup phase............................. 37

Figure 4.2 EGT deviation indicator in starter healthy condition ........................... 41

Figure 4.3 EGT deviation indicator in starter faulty condition............................. 42

Figure 4.4 Starter health indicator in starter healthy condition.............................. 43

Figure 4.5 Starter health indicator in starter faulty condition ............................... 44

Figure 5.1 PDF and CDF of failures with respect to the EGT deviation indicator.... 49

Figure 5.2 PDF and CDF of failures with respect to the starter health indicator....... 50

Figure 5.3 Implementation of the EGT deviation indicator for diagnosis ................ 51

Figure 5.4 Implementation of the starter health indicator for diagnosis .................. 52

Figure 5.5 Bivariate histogram for both indicators .......................................... 53

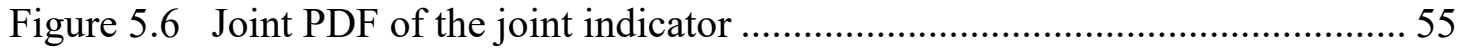


Figure 5.7 Joint CDF of the joint indicator ................................................. 56

Figure 5.8 Implementation of the joint indicator for diagnosis ............................57

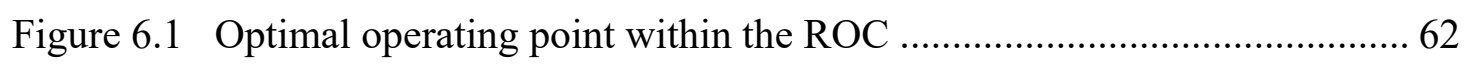

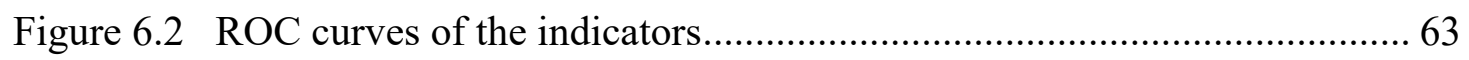

Figure 6.3 Expected costs at various fault detection probability ........................... 65 


\section{List of Symbols}

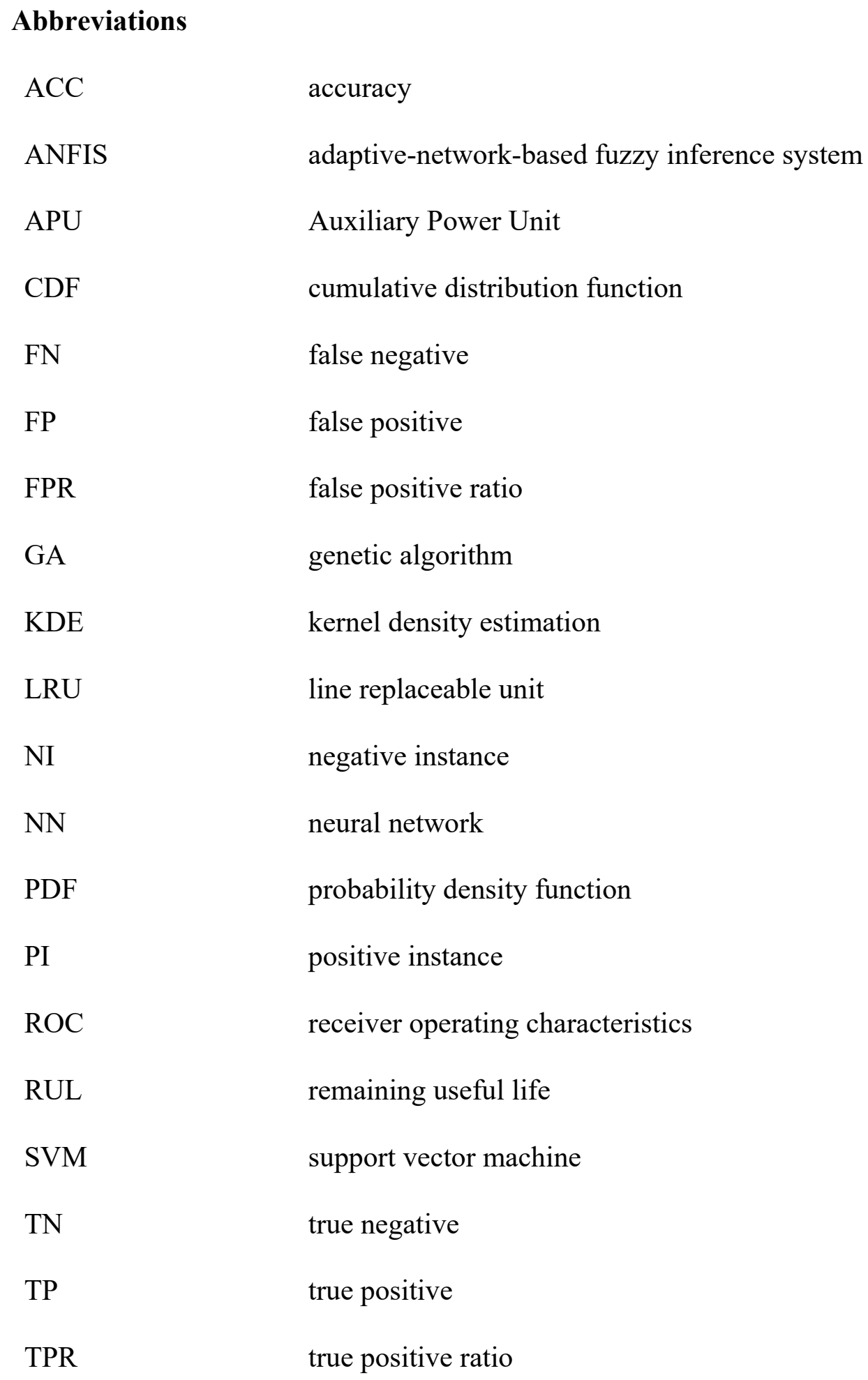




\section{Notation}

\begin{tabular}{|c|c|}
\hline$a_{d}$ & acceleration decline factor \\
\hline C & constant \\
\hline$C_{p}$ & specific heat at constant pressure \\
\hline$E G T$ & Exhaust Gas Temperature \\
\hline$\hat{f}$ & density estimator \\
\hline$F$ & mathematical function \\
\hline $\mathrm{H}$ & bandwidth matrix \\
\hline$b$ & bandwidth \\
\hline$h$ & specific enthalpy \\
\hline$i$ & net input \\
\hline$I$ & health indicator \\
\hline$J$ & moment of inertia \\
\hline$K$ & factor \\
\hline$K E$ & kernel smoothing function \\
\hline$m$ & mass flow \\
\hline$n$ & sample size \\
\hline$N$ & shaft speed \\
\hline$R$ & correlation coefficient \\
\hline$P$ & total pressure \\
\hline$P_{a m b}$ & ambient pressure \\
\hline$P R$ & pressure ratio \\
\hline$P R^{*}$ & critical pressure ratio \\
\hline
\end{tabular}




\begin{tabular}{|c|c|}
\hline$P W$ & power \\
\hline$S$ & slope \\
\hline$t$ & start time \\
\hline$T$ & total temperature \\
\hline$T_{a m b}$ & ambient temperature \\
\hline$X_{i}$ & random bivariate samples \\
\hline$x_{i}$ & random sample \\
\hline$Y$ & measured data \\
\hline$\hat{Y}$ & estimated values \\
\hline$\alpha$ & angular acceleration \\
\hline$\gamma$ & ratio of specific heats \\
\hline$\eta$ & isentropic efficiency \\
\hline$\eta_{\infty}$ & polytropic efficiency \\
\hline$\Phi$ & flow coefficient \\
\hline$\psi$ & work coefficient \\
\hline$\omega$ & angular velocity \\
\hline
\end{tabular}

\section{Subscripts}

0

1

2

3 stagnation value

compressor inlet

combustor inlet

turbine inlet 
4

$a$

C

cal

des

$g$

$\max$

mech

peak

$S$

$t$ turbine outlet

air

compressor

calculated

design point

combustion gases

maximum

mechanical

peak point

starter

turbine 


\section{Chapter: Introduction}

\subsection{Overview}

Auxiliary power unit (APU), a small gas turbine, provides pressurized air to start the main engines and electricity for an aircraft before the main engines start [1], [2]. The conventional maintenance strategy applied in the gas turbine industries is based on preventative maintenance [3], [4]. It includes inspections and maintenance actions at a pre-established schedule, neglecting the individual health conditions of the machinery parts. Preventative maintenance possibly increases both the maintenance and the operational costs, due to spending time and money for replacing the parts with residual lives. At the same time, this maintenance strategy does not address the risk of failures incurring between maintenance intervals caused by varying environment or complex operating conditions [5]. With the advancement of sensing and software techniques, condition-based maintenance (CBM) has been more widely adopted in different disciplines, including the gas turbine industry [6]. CBM is based on constant assessment of the machine health condition and optimization of the allocation of maintenance resources for individual situations of the machinery. It can lead to considerable savings, and improvement of availability and safety [7], [8].

Diagnostics is an integral part of CBM, which provides health information of the machine to support CBM. A classical diagnostic approach is to employ techniques to predict the machine performance as the baseline, assuming the machine is in the healthy condition. The predicted values are then compared to the measurement data. The discrepancy between the predicted value and measured data can be extracted as the 
feature to monitor the health condition of the machine and provide indications for the performance deterioration and malfunction prediction [6], [9], [10]. The main techniques to predict the machine performance can be categorized as physics-based model and datadriven method.

To start the APU, an electric motor, also known as the starter, is used. The starter provides the required power to accelerate the rotor to the designated speed to enable the ignitor to light up the APU. With parts wearing and aging, the starter gradually degrades and provides less power than its designated level. The degradation of the starter causes the start time of the APU to get longer and eventually, the APU fails to start. Failing to start the APU is extremely dangerous for an aircraft during a flight, when engines need a restart. Starter failure has been identified as the primary reason for the APU starting failures [11], [12]. A starter failure can also cause operational interruptions with escalated maintenance costs. Therefore, it is essential to predict the health condition of the starter and conduct the corresponding CBM.

To address this problem, this thesis focuses on the development of a physics-based diagnostic scheme to conduct quantitative performance assessment of the starter and provide indications for starter fault diagnostics to support the corresponding CBM.

\subsection{Literature Review}

This section reviews two main techniques used within gas turbine diagnostics: physics-based model and data-driven method. The state-of-the-art related to the APU starter fault diagnostics is also addressed. 


\subsubsection{Physics-based Model for Diagnostics}

As the starter degradation or fault affects the performance of the APU, investigation of the APU behaviors is a good point to start with. APU is a small-scale gas turbine, so the physics laws of gas turbines also apply to the APU. This subsection focuses on the physics-based model of the gas turbine.

Many physical models have been developed to study the gas turbine [13]-[16]. These models adopted aerodynamics and thermodynamics to simulate the gas turbine behaviors. The most popular physics-based model is called Gas Path Analysis (GPA) first introduced by Urban [17]-[19]. As the condition of the gas turbine is reflected by the measurable gas-path parameters, GPA tries to find aero-thermodynamic links between the gas turbine and parameters to assess the gas turbine at both system level and component level [7], [20], [21]. There are mainly two categories within GPA: linear and non-linear GPA models.

Urban utilized linear GPA models to predict the performance of the gas turbine for the first time. Since then, many derivations have been developed to advance the predictive accuracy, i.e. adaptive GPA [15].

Due to the non-linear nature of the gas turbine, non-linear GPA models present more accurate prediction, especially in the off-design conditions. Saravanamuttoo contributed enormously to the development of the non-linear GPA models of the gas turbine [22][26]. Different derivations are developed to study varieties of problems within the gas turbine. Non-linear GPA models have been widely used in both steady condition and transient condition. 
In the steady condition, work compatibility of the gas turbine is assumed when constructing models. That means turbine output work is equal to the work absorbed by the compressor. There is no additional work to accelerate the gas turbine and the shaft speed is considered constant. Houman et al. developed a thermodynamic model for the gas path and applied the model to an industrial gas turbine to predict both short-term recoverable and long-term non-recoverable degradation level of the engine in the steady condition [27]. In this model, the detailed compressor and turbine characteristics information should be available in advance. However, such information is only available to manufacturers, and it is very difficult for users, like airlines, to access.

Some models without component characteristic maps are also established to approximate the gas turbine performance in the steady condition. Saravanamuttoo et al. developed a mathematical model to predict the off-design performance of turbofans [28]. This approach did not need characteristic maps and just required the design point parameters. The turbine in this approach was always assumed to be choked. Suraweera et $a l$. started the simulation with the turbine in the modeling and tried to match the pressure ratio within the compressor, aiming to find off-design operation point [29]. His approach also did not need characteristic maps and assumed the turbine is choked. Wittenberg et al. developed a method by matching mass flow between the turbine and the nozzle. Based on that, the method instituted the overall model for both the compressor and the turbine [30].

In the transient condition, the torque required by the compressor is different from the torque released by the turbine. A part of the net torque is used to accelerate or decelerate the rotor. Also kinetic energy should be involved in the modeling. 
With characteristic maps of components, Saravanamuttoo et al. simulated the transient performance of a single-spool turbojet engine [31]. Kim et al. derived mathematical models by using unsteady one-dimensional governing equations [32]. This model was employed to simulate the transient behavior of a 150MW industrial engine.

Agrawal and Yunis et al. developed a method to estimate transient performance without a need to acquire detailed component characteristics. This method requires some reference values to estimate gas turbine performance within overall operating speed. However, this method must obtain amounts of empirical parameters and curves for some specific cases and such empirical values are not easy to obtain [33].

Some dynamic models are also generated, which focus on investigating the response and control of the gas turbine rather than the gas turbine performance during the transient phase [34], [35].

Physics-based models require a few data to construct the model. Such models have tangible physical meanings and provide more accurate results than other techniques. However, the construction of models always needs deep understanding of the machine and amounts of computational effort. As the architecture of the gas turbine is unique case by case, the adaptation of models is another problem [36].

\subsubsection{Data-driven Method for Diagnostics}

Physics-based models for the complex gas turbines are not always available due to little information or without detailed characteristic maps. Data-driven methods are introduced to address such challenges. For data-driven methods, physical relationships of the machinery are not necessary and quantitative relationships are significant. In the 
viewpoint of mathematics, data-driven methods try to establish a quantitative model to accommodate the relationship between varieties of parameters [6]. Major data-driven methods applied in the gas turbine diagnostics include genetic algorithms (GA), neural networks (NN), fuzzy-logic, adaptive-network-based fuzzy inference systems (ANFIS) and support vector machines (SVM).

GA is an optimization tool, which is theoretically similar to the non-linear physicsbased model. GA first obtains the component parameters of the gas turbine and then predicts gas-path parameters. To obtain optimal results, GA minimizes the difference between predicted values and measurements [37]. Zedda et al. employed GA to estimate the performance for a two-spool, low bypass ratio turbofan, the EJ200. [38], [39]. The estimation is performed through the optimization of an objective function and a high level of accuracy is achieved.

$\mathrm{NN}$ is a parallel computational network that mimics the learning process of the human brain. It performs as a non-linear estimator, mapping input space to output space. Dietz pioneered the use of neural networks into the diagnosis of the gas turbine [40]. A wide range of different neural networks have been applied for gas turbine diagnosis [41]-[44].

Fuzzy-logic is a rule-based approach that formalizes imprecise human reasoning. It provides a multi-input-output system to establish complex functional relationships based on the formulation of an algebra [6], [45], [46]. Fuzzy-logic has been effectively applied for the fault diagnosis and isolation within the gas turbine [47]-[50]. Eustace employed fuzzy logic in a real-world application with eight example data. The disadvantages of attributing measurement noise or sensor errors to changes in engine condition is overcome by this method. 
ANFIS is the integration of both fuzzy logic and neural networks. This method benefits from the advantages of both approaches. Its architecture can be constructed based on both human knowledge and data pairs of inputs and outputs [51], [52]. Hanachi et al. applied ANFIS to a gas turbine under diverse ambient conditions and control settings. The results prove the competency of the ANFIS framework in the fault diagnosis.

SVM is a classifier that uses hyperplanes in the high-dimensional data space for classification [6], [53], [54]. Zhou et al. utilized SVM to diagnose a deteriorated gas turbine. Training and testing were implemented during the application of SVM and a total eight types of faults within the component level were identified. The diagnostic accuracy can reach $91.67 \%$ [55].

Data-driven methods are always needed when physics-based models are not available for some complex machines. No expert knowledge of the machinery is required in advance. However, their processes lack of transparency and are opaque to the users. Massive amounts of data are required for the training of the models [9].

\subsubsection{Previous Works on the Starter Fault Diagnostics}

The startup phase is regarded as one of the most technically challenging aspects when modeling and simulating gas turbine performance [56]. Some efforts have been conducted to reveal this process and diagnose starter failure. Kim et al. applied multiple performance parameters for degradation monitoring and diagnosis of the starter. Deterioration of the performance parameters were observed to occur sequentially and the failure was detected based on measurements within multiple time steps [57]. Lou 
employed measurements from the APU sensors as indicators for the starter health monitoring and failure detection [58]. However, since the monitored performance parameters were selected at the system level, the results could potentially be affected by degradation of the other components of the APU, and are not restricted to the effects of the starter faults. Yang et al. defined the threshold of the indicator at a fixed number of fault detection probability, i.e., 95\% [59]. The limitation is that, a fixed level of the fault detection probability is not necessarily the optimum answer for the diagnostic performance. The optimum threshold depends on variable factors such as the failure risk and the maintenance costs. Kim et al. defined the thresholds of multiple indicators in several one-dimensional feature spaces, where the thresholds were set independently [12]. It is very challenging to decide if a failure occurs when only one indicator exceeds its corresponding threshold while the others do not.

Most diagnostic approaches in the literature are based on statistical analysis and datadriven techniques [12], [57]-[60]. These approaches cannot quantitatively identify the health level of the starter. Subjective judgment is involved during verification of the data related to the starter fault. That definitely affects the decision-making and introduces uncertainty and safety risk. Also, these approaches significantly depend on historical data of measurements and require substantial amounts of measured data to assess the health condition of the starter. With only one measured point of data, these methods cannot provide desired results. That limits the range of applications. Finally, the process of these approaches is opaque to users and it is very difficult to reveal the physical meanings of the failure. 


\subsection{Thesis Objectives}

An electric starter provides the initial power to run the APU during the startup process. Degradation of the starter affects the APU starting performance and even causes the APU to fail to start. To address this challenge, a physics-based diagnostic framework for the starter failure within APUs is developed in this thesis with following objectives.

1. To develop a generic physics-based model for APU starting process without a need to require detailed characteristic maps of components. Performance deterioration is monitored by comparing predicted values from the established model to the measured data.

2. To develop some health indicators to quantitively predict the degradation severity and the health condition of the starter, providing symptoms for the starter failures.

3. To develop an enhanced joint indicator that employs multi-dimensional feature spaces to enable multiple-feature diagnosis for the starter.

4. To develop a scheme to evaluate the diagnostic performance of the health indicators based on the trade-off between the diagnostic reliability and the operating cost reduction.

5. To establish a diagnostic framework for the starter failure to provide substantial information to support the corresponding CBM. 


\subsection{Experimental Data}

The data in this study are collected from a fleet of Airbus 310 routine records. The APU used in this study is GTCP 331, manufactured by Honeywell [61]. The data includes 14 cases with starter healthy condition and 11 cases with starter faulty condition. Five parameters are recorded during each APU starting cycle, including APU starting time $(t)$, peak Exhaust Gas Temperature $\left(E G T_{\text {peak }}\right)$, shaft speed at peak $E G T$ point $\left(N_{\text {peak }}\right)$, ambient temperature $\left(T_{a m b}\right)$, and the altitude that can be used to estimate the ambient pressure $\left(P_{a m b}\right)$.

The operating parameters of the APU at the design point are available, as provided in Table $1.1[61]$.

Table 1.1 Parameters of APU at the design point

\begin{tabular}{|l|c|}
\hline Parameters & Values \\
\hline Rotational Speed, rpm & 40,400 \\
\hline Corrected Inlet Airflow, kg/s & 3.0 \\
\hline Turbine Inlet Temperature, ${ }^{\circ} \mathrm{C}$ & 981 \\
\hline Compressor Pressure Ratio & $8.7: 1$ \\
\hline Output Shaft Horsepower, $\mathrm{kW}$ & 430 \\
\hline Fuel Consumption, $\mathrm{kg} / \mathrm{h}$ & 173.5 \\
\hline
\end{tabular}




\subsection{Thesis Outline}

The rest of the thesis is organized as follows.

Chapter 2 lays out a thermodynamic model associated with the transient operation of the APU during the starting period. The physical link between APU performance parameters and the starter degradation is revealed.

For the comparative study, a back-propagation, feedforward neural network model is structured, trained and tested in Chapter 3. The network architecture, the training algorithm and generalization are presented. The performance assessment of APU based on both physics-based model and neural network model are exhibited and compared.

In Chapter 4, two individual health indicators are derived from the physics-based model for the starter health monitoring. Implementation results of both indicators are presented and a comparison of the two individual indicators are discussed.

Chapter 5 presents the diagnostics using individual health indicators and proposes an enhanced joint indicator for the starter fault diagnostics to improve diagnostic performance.

In Chapter 6, ROC is introduced to evaluate the diagnostic performance of the three indicators. The optimal threshold is determined based on balance between benefits and costs. The expected cost of three indicators are analyzed and compared.

A conclusive summary and recommendations for the future work are provided in Chapter 7. 


\section{Chapter: Physics-based Model for APU Performance Monitoring}

\subsection{Overview}

In large aircrafts, an APU, which is usually a small gas turbine is utilized to start the main engines and provide the required power when the engines are off. During the APU startup phase, an electric motor called the starter, is used to provide initial power for running the gas turbine. With degradation of the starter, its mechanical power to accelerate the gas turbine decreases and eventually, the APU fails to start. This can cause serious consequences and potential safety hazards. Therefore, it is necessary to monitor the starter health condition and provide substantial information to conduct CBM for the starter.

In most APUs in service, the existing sensors are mounted for system control rather than the health monitoring [62]. Since the starter is connected to the APU and provides the initial power, performance of the APU and the corresponding measurements are affected during the startup process if the starter gets faulty. Therefore, measurements from the existing sensors of the APU are the only readily available data to monitor the health condition of the starter.

Some previous works in the literature attempt to estimate the performance of the starter based on statistical analysis and data-driven methods [63], [64]. These approaches lack transparency and do not possess tangible physical meanings [58], [60], [65]. They also significantly depend on historical measurement data.

To the best of the author's knowledge, a physics-based approach has not been adopted to study the starter health condition. In this chapter, a novel physics-based approach is 
developed for health monitoring of the starter, which involves both the starter and the APU and considers kinetic energy during the startup phase. This approach adopts mathematical models with no need to access the detailed component characteristics. The Buckingham PI theorem is employed to generate the compressor model whereas a characteristic curve of the turbine is adopted to approximate the turbine performance. With flow compatibility and energy conservation, the physics-based model of the APU for the startup phase is constructed. Using a few measured data points, the model can be calibrated for performance prediction of the APU.

The APU model in this study is a GTCP 331 gas turbine. It is a single-spool small gas turbine, which includes a two-stage centrifugal compressor, an annular combustion chamber, and a three-stage axial turbine [1], [61]. Figure 2.1 shows the thermodynamic cycle of the APU, where the label 1 means the inlet of the compressor; the label 2 means the outlet of the compressor and the inlet of the combustion chamber; the label 3 means the outlet of the combustion chamber and the inlet of the turbine; the label 4 means the outlet of the turbine.

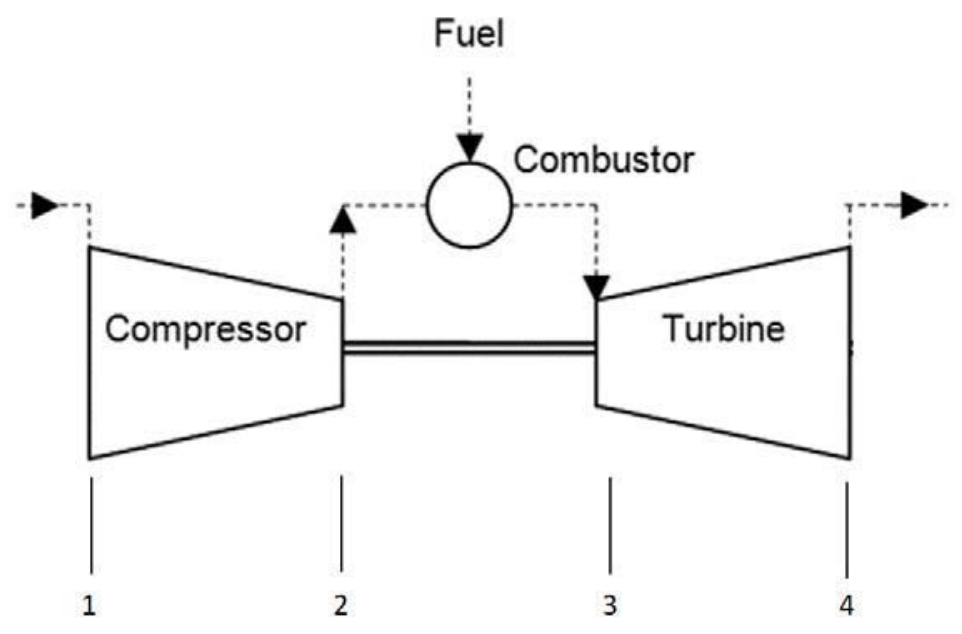

Figure 2.1 Thermodynamic cycle of the APU. 


\subsection{Compressor Model}

The Buckingham PI theorem is used to establish the mathematical model of the compressor, which is a kind of dimensional analysis [33], [66], [67]. This method adopts normalized forms of parameters to relate performance at both reference speed and other operating speeds. The design point parameters of the gas turbine are available, which are used as the reference values.

Mass flow in the inlet of the compressor $m_{1}$ has the following relation in both the transient condition and the design point [33].

$$
\frac{\frac{m_{1} \sqrt{T_{01}}}{P_{01}}}{\left(\frac{m_{1} \sqrt{T_{01}}}{P_{01}}\right)_{d e s}}=\frac{\left(\frac{N}{\sqrt{T_{01}}}\right)}{\left(\frac{N}{\sqrt{T_{01}}}\right)_{d e s}} \times \frac{\Phi}{\Phi_{d e s}}=K_{\Phi} \frac{\left(\frac{N}{\sqrt{T_{01}}}\right)}{\left(\frac{N}{\sqrt{T_{01}}}\right) d e s}
$$

where, $T_{01}$ and $P_{01}$ are the compressor inlet temperature and pressure respectively, $N$ is the shaft speed, $\Phi$ is the flow coefficient and des means the parameters in the design point. The value of flow coefficient factor $K_{\Phi}$ is determined empirically. To obtain a suitable $K_{\Phi}$ value, generalized centrifugal compressor characteristics that cover low speed and startup phase are used to generate the specific curve for the model in the study [68]. Figure 2.2 shows a typical curve for the $K_{\Phi}$ value.

The compressor work can be presented in a similar way.

$$
\frac{\frac{\Delta h_{c}, \text { actual }}{T_{01}}}{\left(\frac{\Delta h_{c}, \text { actual }}{T_{01}}\right)_{\text {des }}}=\frac{\left(\frac{N}{\sqrt{T_{01}}}\right)^{2}}{\left(\frac{N}{\sqrt{T_{01}}}\right)_{d e s}^{2}} \times \frac{\psi}{\psi_{\text {des }}}=K_{\psi} \frac{\left(\frac{N}{\sqrt{T_{01}}}\right)^{2}}{\left(\frac{N}{\sqrt{T_{01}}}\right)_{d e s}^{2}}
$$


where $\Delta h_{c}$, actual is the actual enthalpy increase, $\psi$ is the work coefficient, and $K_{\psi}$ is the work coefficient factor.

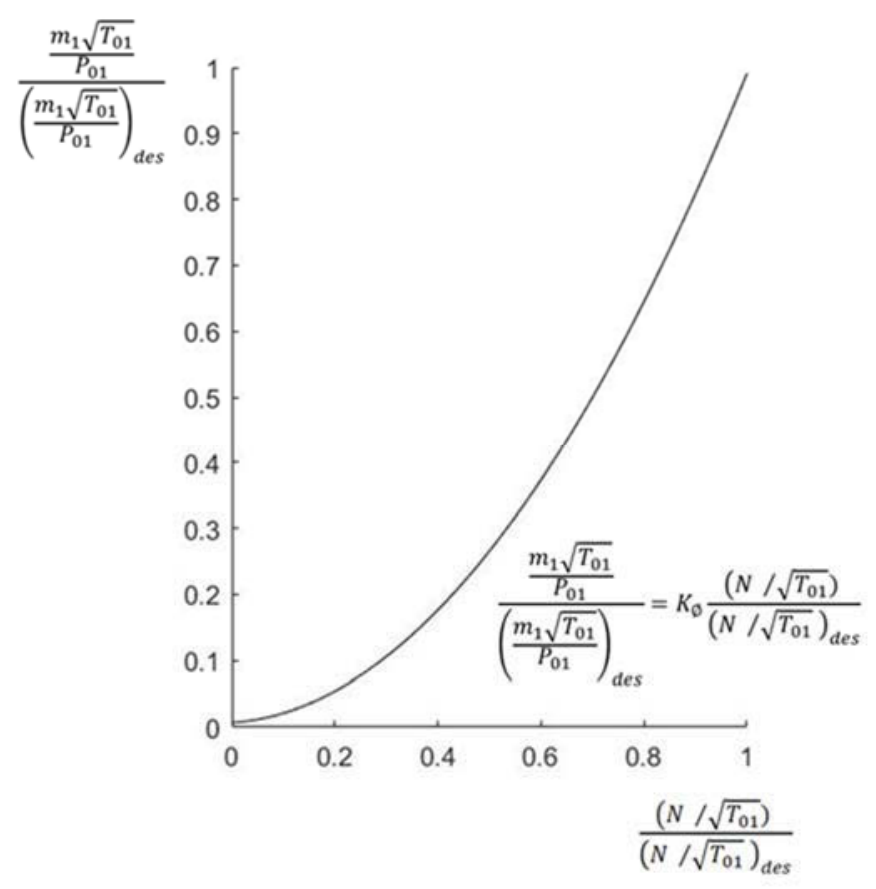

Figure 2.2 The typical curve for $K_{\Phi}$.

Based on thermodynamics for the compressor, the pressure ratio of the compressor $P_{02} / P_{01}$ has following relationship with enthalpy.

$$
\begin{aligned}
& \eta_{c}=\frac{\Delta h_{c}, \text { ideal }}{\Delta h_{c}, \text { actual }}, \\
& \Delta h_{c}, \text { ideal }=C_{p} \times T_{01} \times\left[\left(\frac{P_{02}}{P_{01}}\right)^{\frac{\gamma-1}{\gamma}}-1\right],
\end{aligned}
$$


where $\eta_{c}$ is the isentropic efficiency of the compressor, $\Delta h_{c}$, ideal is the ideal enthalpy increase, $C_{p}$ is the specific heat at constant pressure, and $\gamma$ is the ratio of specific heats.

With Eqs (2.2), (2.3) and (2.4), the pressure ratio of the compressor at both the operating point and the design point are related, as shown in Eq (2.5)

$$
\frac{\left[\left(\frac{P_{02}}{P_{01}}\right)^{\frac{\gamma-1}{\gamma}}-1\right]}{\left[\left(\frac{P_{02}}{P_{01}}\right)^{\frac{\gamma-1}{\gamma}}-1\right]_{d e s}} \times \frac{1}{\frac{\eta_{c}}{\left(\eta_{c}\right)_{d e s}}}=K_{\psi} \frac{\left(N / \sqrt{T_{01}}\right)^{2}}{\left(N / \sqrt{T_{01}}\right)_{d e s}^{2}}
$$

Also, isentropic efficiency $\eta_{c}$ and polytropic efficiency $\eta_{\infty c}$ of the compressor have the following relation [23].

$$
\begin{aligned}
& \eta_{c}=\frac{\left(P_{02} / P_{01}\right)^{\frac{\gamma-1}{\gamma}}-1}{\left(P_{02} / P_{01}\right)^{\frac{\gamma-1}{\gamma \times \eta_{\infty c}}}-1} \\
& \eta_{c}=F\left(\eta_{\infty c}\right) .
\end{aligned}
$$

Substituting Eq. (2.6) into Eq. (2.5), the equation has the following expression.

$$
\frac{\left[\left(\frac{P_{02}}{P_{01}}\right)^{\frac{\gamma-1}{\gamma \times \eta_{\infty c}}}-1\right]}{\left[\left(\frac{P_{02}}{P_{01}}\right)^{\frac{\gamma-1}{\gamma \times \eta_{\infty c}}}-1\right]_{d e s}}=K_{\psi} \frac{\left(N / \sqrt{T_{01}}\right)^{2}}{\left(N / \sqrt{T_{01}}\right)_{d e s}^{2}}
$$


Work coefficient factor $K_{\psi}$ shows a functional relationship with $K_{\Phi}$ [23], [69], [70].

$$
K_{\psi}=F\left(K_{\Phi}\right)
$$

Polytropic efficiency of the compressor $\eta_{\infty c}$ during the startup phase can be determined by the polytropic efficiency factor $K_{\eta \infty_{c}}$, which also happens to be a function of $K_{\Phi}[71]$

$$
K_{\eta_{\infty c}}=\eta_{\infty c} /\left(\eta_{\infty c}\right)_{d e s}=F\left(K_{\Phi}\right)
$$

Then, the pressure ratio of the compressor $P_{02} / P_{01}$ in the transient condition can be calculated as a function of shaft speed $N$, compressor inlet temperature $T_{01}$ and pressure $P_{01}$

$$
P_{02} / P_{01}=F\left(K_{\psi}, K_{\eta_{\infty c}}, N, T_{01}, P_{01}\right)=F\left(N, T_{01}, P_{01}\right)
$$

As a result, corrected mass flow, pressure ratio, and efficiency of the compressor can be calculated based on the corrected shaft speed.

\subsection{Turbine Model}

Shaft speed has little effect on the mass flow of the turbine and the mass flow mainly relies on pressure ratio across the turbine [22], [30]. Characteristic curve of the turbine that establishes the relationship between the corrected mass flow and pressure ratio is approximated as [30]: 


$$
\left(\frac{m_{3} \sqrt{T_{03}}}{P_{03}}\right)^{2}=C \times\left[\left(1-\frac{1}{P R^{*}}\right)^{2}-\left(\frac{1}{P R_{t}}-\frac{1}{P R^{*}}\right)^{2}\right]
$$

With design point parameters, the constant $(C)$ in Eq. (2.11) can be determined. A typical value of the critical pressure ratio (at which choking is attained) for a three-stage turbine is $P R^{*}=3$, see Fig. 2.3 [72], [73].

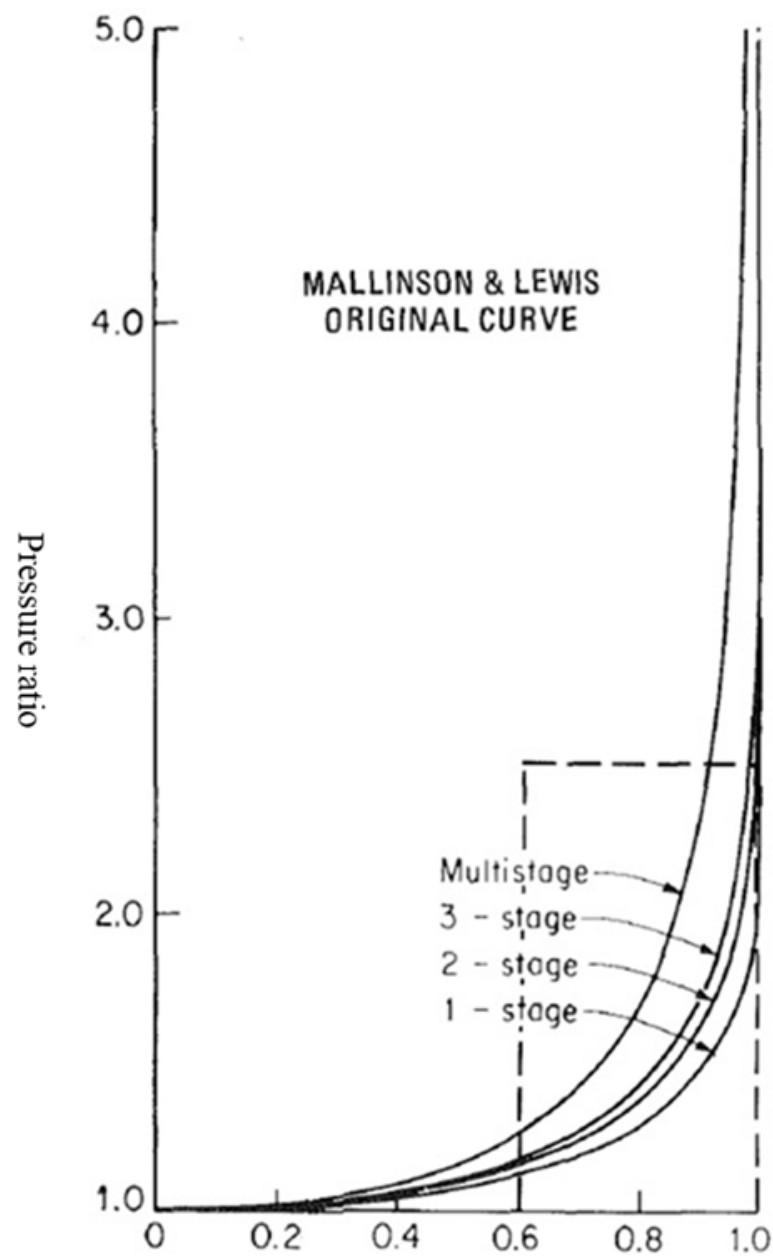

Relative inlet mass flow coefficient

Figure 2.3 Critical pressure ratio in approximate turbine characteristics [72] 
The turbine pressure ratio $P R_{t}$ can be determined through Eq. (2.12) when the compressor ratio $\left(P_{02} / P_{01}\right)$ is specified.

$$
P R_{t}=\frac{P_{03}}{P_{04}}=\frac{P_{03}}{P_{02}} \times \frac{P_{02}}{P_{01}} \times \frac{P_{01}}{P_{04}}
$$

Inlet and exhaust pressure losses are neglected in the estimation; therefore $P_{01} / P_{04}=$ 1. Combustion chamber pressure ratio $\left(P_{03} / P_{02}\right)$ is determined by the chamber pressure loss, which is estimated as a fraction of the compressor delivery pressure. Therefore, the combustion chamber pressure ratio is assumed constant in most simulations [23]. In this way, the corrected mass flow and pressure ratio of the turbine are determined.

\subsection{Transient Model}

Flow compatibility and energy conservation are the bases to establish the transient model of the APU.

\subsubsection{Flow Compatibility}

Flow compatibility holds in both steady state and transient condition with slow variations of shaft speed [74]. Mass flow through the turbine is assumed to be equal to that through the compressor. Nevertheless, in reality, air leakage and the added fuel flow slightly change this equation. Assuming the mass flow of air leakage equal to the fuel flow [23], corrected mass flow of the turbine and the compressor form the following equation. 


$$
\frac{m_{3} \sqrt{T_{03}}}{P_{03}}=\frac{m_{1} \sqrt{T_{01}}}{P_{01}} \times \frac{P_{01}}{P_{02}} \times \frac{P_{02}}{P_{03}} \times \frac{\sqrt{T_{03}}}{\sqrt{T_{01}}}
$$

\subsubsection{Energy Conservation}

During the transient condition, a part of the mechanical work is absorbed for accelerating the gas turbine rotor. In this condition, the energy conservation equation includes the turbine power $P W_{t}$, the compressor power $P W_{c}$, the starter power $P W_{s}$ and variation of kinetic energy:

$$
P W_{t}-\frac{1}{\eta_{\text {mech }}} P W_{c}+P W_{s}=J \omega \alpha
$$

where $\eta_{\text {mech }}$ is the mechanical efficiency, $J$ is moment of inertia, $\omega$ is angular velocity and $\alpha$ is rotor acceleration.

Acceleration $\alpha$ can be estimated using the simulated data of APU [62], [75].

$$
\alpha=F(N)
$$

Output power of the starter depends on the shaft speed [33], [56]. It is estimated based on the starter maximum power from the product specification catalogue.

$$
P W_{s}=F\left(P W_{s, \max }, N\right)
$$


$\eta_{\text {mech }}$ is the mechanical efficiency of the compressor-turbine combination. Mechanical losses are mainly contributed by frictional loss in bearings and gears, and aerodynamic windage loss in the compressor and turbine. It usually accounts for less than $5 \%$ of the compressor power [33].

In Eq. (2.14), power of the compressor is a function of compressor efficiency and pressure ratio [23].

$$
\begin{aligned}
& P W_{c}=m_{1} \times C_{p a} \times\left(T_{02}-T_{01}\right), \\
& T_{02}-T_{01}=\frac{T_{01}}{\eta_{c}} \times\left[\left(\frac{P_{02}}{P_{01}}\right)^{\frac{\gamma-1}{\gamma}}-1\right] \\
& P W_{c}=F\left(\eta_{c}, P_{02} / P_{01}\right) .
\end{aligned}
$$

And the turbine power can be found as a function of $T_{03}$ and $E G T\left(T_{04}\right)$ [76].

$$
P W_{t}=m_{3} \times C_{p g} \times\left(T_{03}-T_{04}\right)
$$

Value of $T_{03}$ can be calculated by Eq. (2.13), and EGT remains the only unknown of the Eq. (2.14). With rearranging Eq. (2.14), EGT can be calculated as a function of independent variables:

$$
E G T_{c a l}=F\left(T_{01}, P_{01}, N, \alpha, P W_{s}\right)
$$


In this study, APU measurements are obtained from the operating data of a fleet of Airbus A310 aircraft from the existing sensors of the system. During each starting cycle of APUs, five parameters are recorded that include APU starting time $(t)$, peak Exhaust Gas Temperature $\left(E G T_{\text {peak }}\right)$, shaft speed at peak $E G T$ point $\left(N_{\text {peak }}\right)$, ambient temperature $\left(T_{a m b}\right)$, and the altitude that can be used to estimate the ambient pressure $\left(P_{a m b}\right)$.

The physics-based model for APU starting process can be mathematically represented as:

$$
F\left(T_{a m b}, P_{a m b}, N_{\text {peak }}, \alpha, P W_{s}, E G T_{\text {peak }}\right)=0 .
$$

With the measurements of $T_{a m b}, P_{a m b}$ and $N_{\text {peak }}$, and $P W_{s}$ and $\alpha$ in the starter healthy conditions, Eq. (2.19) can be arranged as following.

$$
E G T_{\text {peak }} \text { cal }=F\left(T_{a m b}, P_{a m b}, N_{\text {peak }}, \alpha, P W_{s}\right) .
$$

Figure 2.4 shows the process flow for implementation of the transient model. 


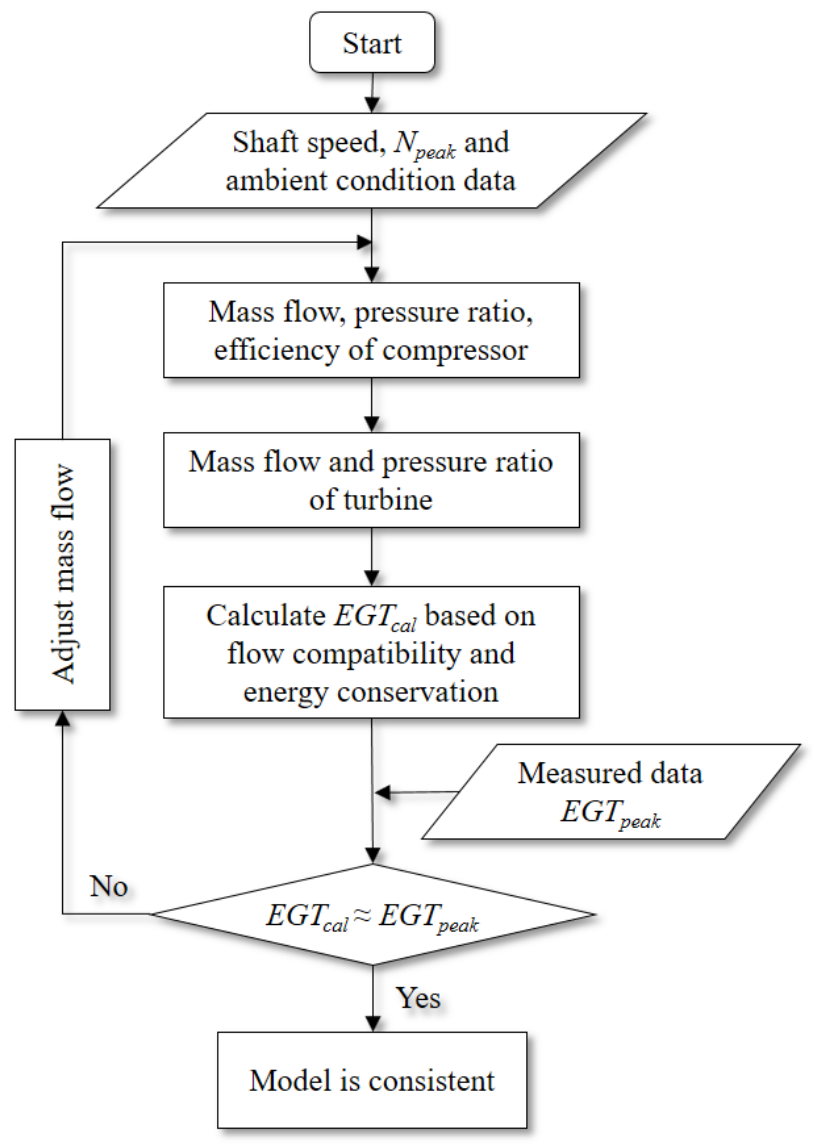

Figure 2.4 Procedures for implementing the transient model

\subsection{Summary}

In this chapter, a physics-based transient model of the gas turbine is established to monitor the performance of the APU startup phase. With limited information on component characteristics and measurement data, the model is constructed based on a generic approach for the gas turbine starting process. The Buckingham PI theorem is used to establish the compressor model while a generalized turbine characteristic curve approximates the performance of the turbine. The model takes into account both the starter and the gas turbine and reveals the physical link between the starter parameters and the APU performance. Using the operating data from the existing sensors of the 
aircraft, the transient model can monitor the health condition of the APU and consequently the starter. The results enable decision making for predictive maintenance management and warrant safe operation of the APU system in aircraft. 


\section{Chapter: Neural Network Model for APU Performance Monitoring}

\subsection{Overview}

Alternative to the physics-based model in Chapter 2, a back-propagation, feedforward neural network is used for APU performance monitoring to compare with the physicsbased results. Previous works [58], [59] have attempted to estimate the starter degrading trend, however a clear symptom for the starter failure is not provided, which could be instrumental for the CBM. Both physics-based model and neural network model are implemented in the healthy and faulty conditions, and their capabilities as monitoring tools for the starter failure are verified. The physics-based approach provides more accurate results for the cases with faulty starters, whereas the neural network model shows superior results with the starters in healthy condition.

\subsection{Neural Network Model}

As an artificial intelligence technique, neural networks are widely used in the diagnostics and prognostics field for gas turbines [77]-[80]. Neural networks possess many features that enable them to solve many complex tasks. The capability of function approximation within the neural network [81], [82] is chosen in this study.

Since the starter is connected to the gas turbine and injects power during the startup phase, it will affect the performance of the APU if it gets degraded. Therefore, the APU gas-path measurements collected from the existing sensors of the aircraft can be used to assess the state of the starter degradation [63], [65]. Exhaust gas temperature ( EGT ) is

measured by the majority of the control systems within gas turbines and it is therefore 
selected as a study object to monitor the health condition of the starter. Based on analysing the physical relationship of the gas-path parameters, measurements on $N_{\text {peak }}$, $T_{a m b}$, and $P_{a m b}$ during each starting cycle are chosen as input vectors to estimate $E G T_{\text {peak }}$. A 3-10-1 multilayer feedforward network is structured, including one input layer, one hidden layer and one output layer, as shown in Fig. 3.1.

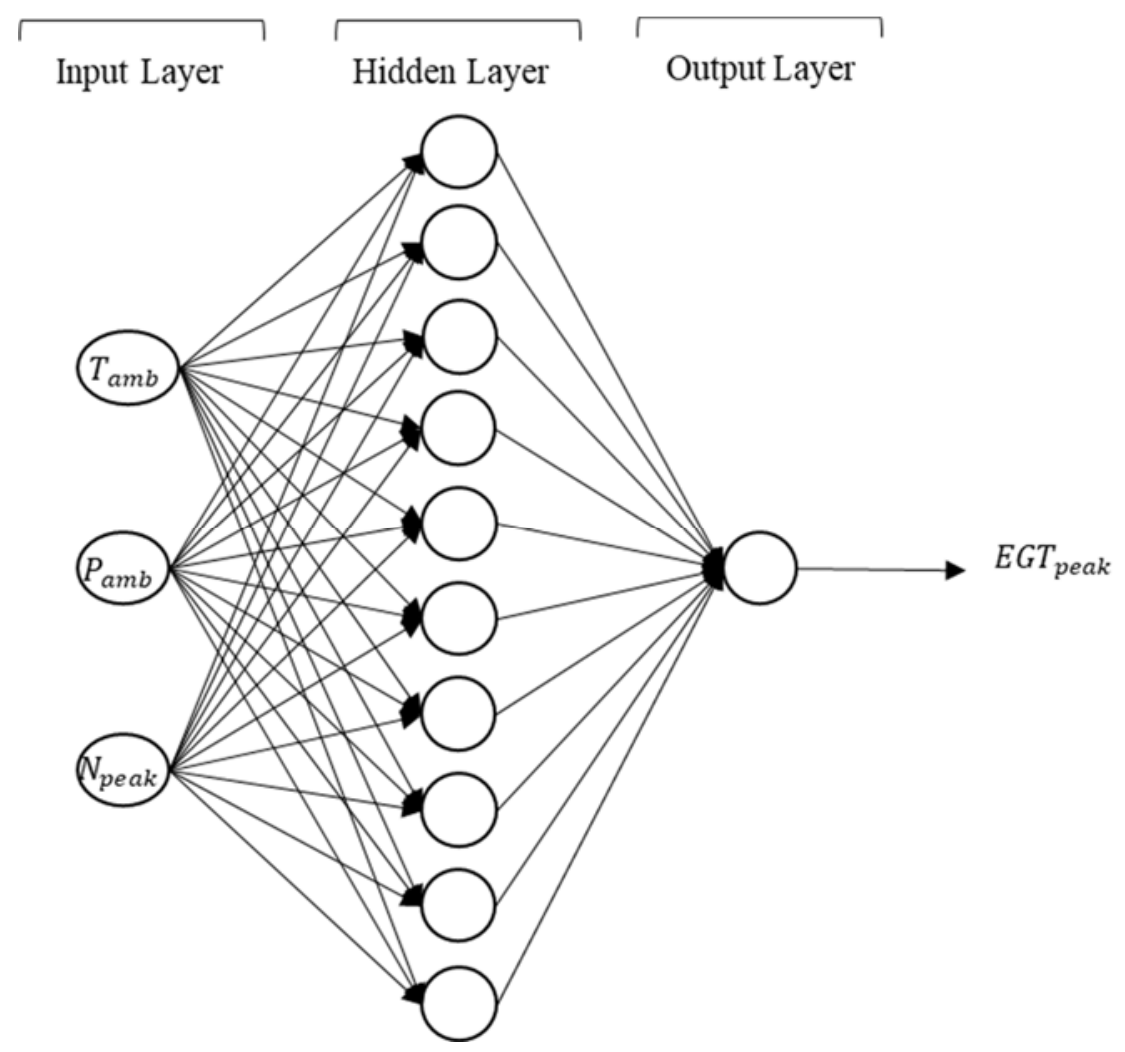

Figure 3.1 The architecture of the 3-10-1 network

Three measured data are utilized as the input vectors for the network, and the numerical output is estimated based on the established network model, which is a nonlinear input-output mapping. Two-layers networks are sufficient for the function approximation within desirable accuracy, provided the hidden layers have enough 
neurons [82], [83]. The hidden layer possesses 10 neurons, in which non-linear hyperbolic tangent sigmoid transfer function is used as shown in Eq. (3.1), which makes the multilayer network suitable for the complex non-linear task, i.e. the function approximation in the study [82].

$$
f(i)=\frac{e^{i}-e^{-i}}{e^{i}+e^{-i}}
$$

A linear transfer function is adopted in the output layer.

The data including 325 measured cycles within a healthy condition are selected to train the network. The Levenberg-Marquardt backpropagation is chosen for learning algorithm [81]. The training stage aims at minimizing the error between estimated values and target values. The training procedures of the feedforward network with backpropagation algorithm are presented in Fig. 3.2. In the forward computation, synaptic weights are fixed, as they have been adjusted during the backward computation [82].

When the network is constructed using the training data, it should still work correctly on the entire population, which is knowns as "generalization" [81]. To achieve such a goal, cross validation is used during the training process. Within the training data, $80 \%$ is used to train the model, $15 \%$ for the validation and $5 \%$ for testing purpose respectively through employing MATLAB neural network toolbox [84].

By this stage, the network is structured, trained and validated. The results of its implementation in both healthy and faulty condition are presented and discussed in the next section. 


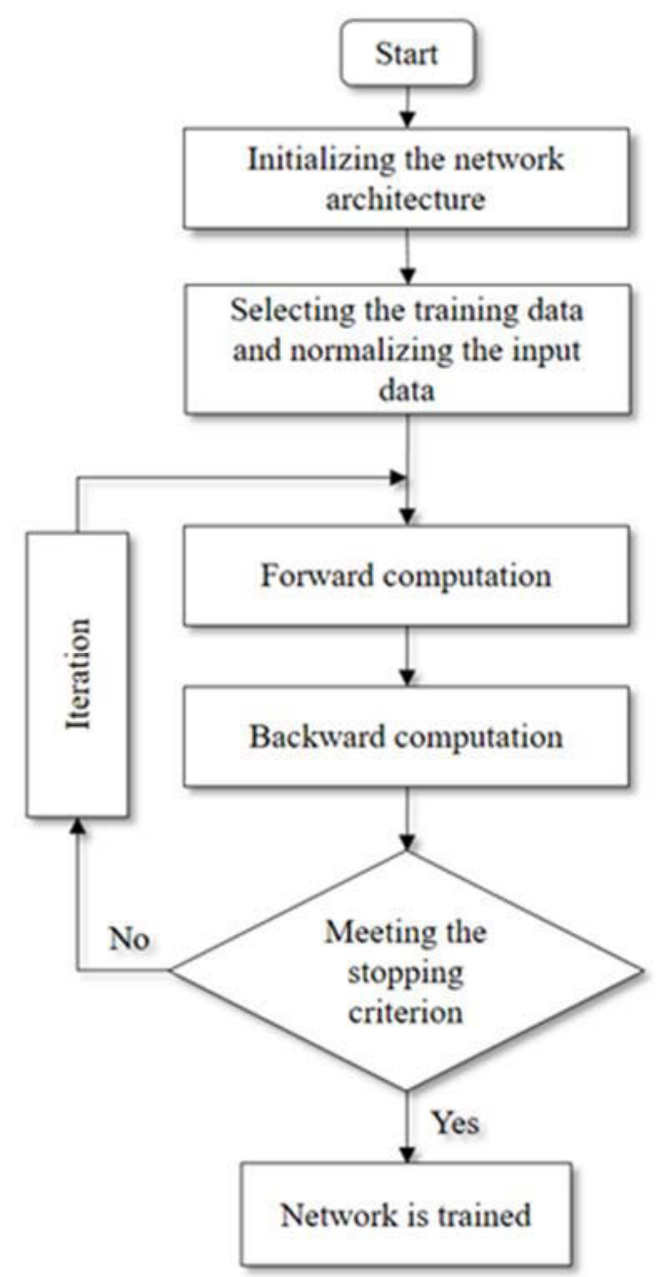

Figure 3.2 Training procedures of the neural network.

\subsection{Implementation in the Starter Healthy Condition}

At first, both physics-based and neural network models are implemented in the healthy condition, where the starter is healthy. Figures. 3.3 and 3.4 show the estimated values beside the measured values for the physics-based and neural network models respectively. The estimated values have a good consistency with the measurements. Both estimated values and measured data vary within a limited range and no rapid trend of long-term changes are shown during the measurement time span. This implies the starter 
has been always in its healthy condition. Both models show their competence to estimate the $E G T_{\text {peak }}$ in a healthy condition. Though some deviations still exist between estimated values and measured data, the variation trend of the $E G T_{\text {peak }}$ is clearly predicted. Considering the lack of characteristic information of the gas turbine, the results are acceptably accurate.

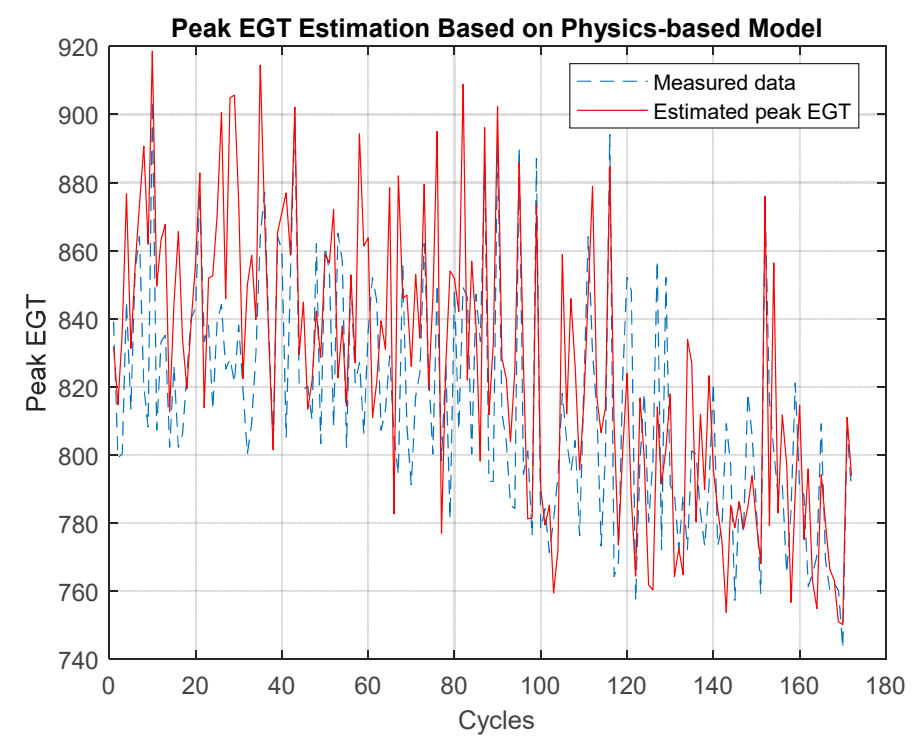

Figure 3.3 Implementation in healthy condition with physics-based model.

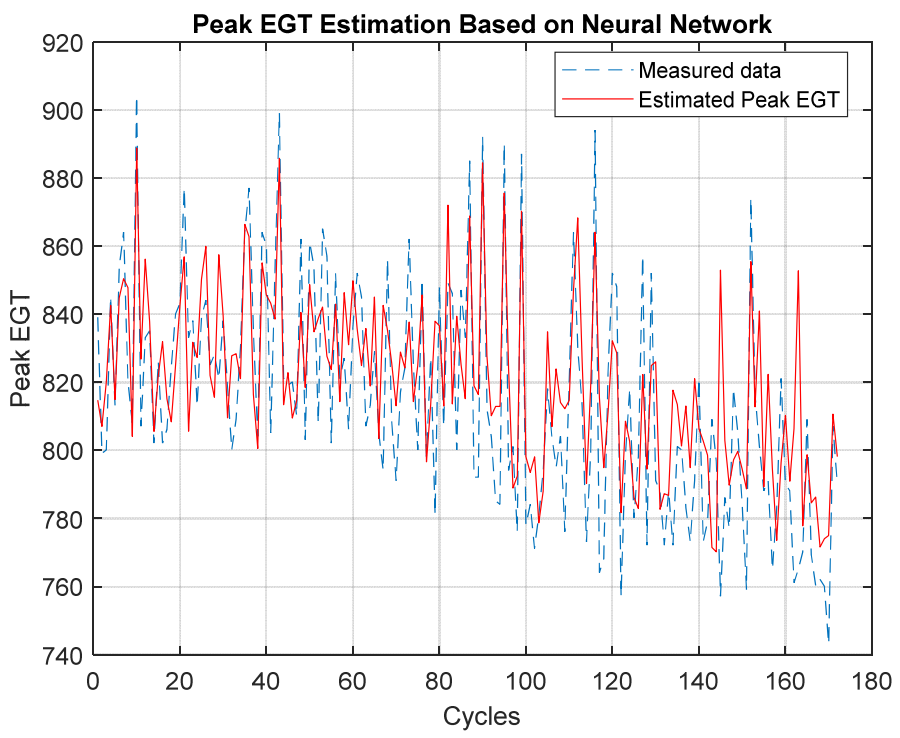

Figure 3.4 Implementation in healthy condition with neural network model. 
Accuracy of the estimated $E G T_{\text {peak }}$ compared to the measured data is different with each model. To quantify the performance of the models, correlation coefficient $(R)$, mean absolute error (MAE) [85], and mean squared error (MSE) [84] are employed. The two latter metrics represent the error between the estimated values $\widehat{Y}_{l}$ and the measured data $Y_{i}$.

$$
\begin{aligned}
\mathrm{MAE} & =\frac{1}{n} \sum_{j=1}^{n}\left|Y_{j}-\widehat{Y}_{J}\right| . \\
\mathrm{MSE} & =\frac{1}{n} \sum_{j=1}^{n}\left(Y_{j}-\widehat{Y}_{J}\right)^{2} .
\end{aligned}
$$

where $n$ is the size of the sample.

The performance results for the starter healthy condition are presented in Table. 3.1, where the neural network model shows less error compared to the physics-based model.

Table 3.1 Performance results of two models

\begin{tabular}{|c|c|c|}
\hline Unit $\left({ }^{\circ} \mathrm{C}\right)$ & Neural network Model & Physics-based Model \\
\hline MAE & 18.33 & 24.19 \\
\hline MSE & 525.6 & 965.1 \\
\hline$R$ & 0.7491 & 0.7078 \\
\hline
\end{tabular}

\subsection{Implementation in the Starter Faulty Condition}

Subsequently, both models are implemented in starter faulty conditions. Distinct patterns are shown in both Fig. 3.5 and Fig. 3.6, in contrast to the results within the starter 
healthy condition. Both estimated values present significant deviations comparing to the measured data at around remaining useful life (RUL) $=125$ in case (a) and RUL $=50$ in case (b). Both models estimate $E G T_{\text {peak }}$ assuming the starter in the healthy condition while the measured data is recorded under faulty conditions of the starter. With the starter getting degraded, its output power for the gas turbine acceleration diminishes while the APU still tries to start within nominal time range. More fuel flow is added through APU control system [74] to compensate the deficiency of the starter power, which causes higher EGT compared to the corresponding values in the healthy condition at the same shaft speed. The gradually increasing deviations between the estimated values and the measured data imply the condition of the starter is deteriorating more and more.

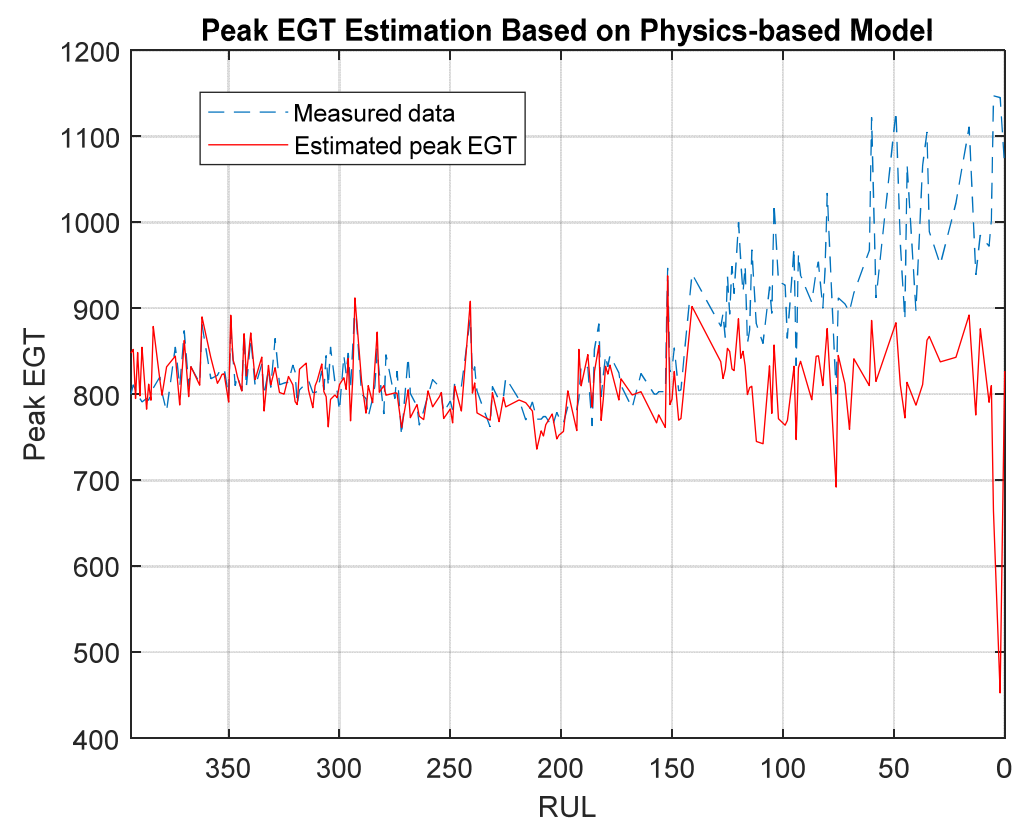

(a) 


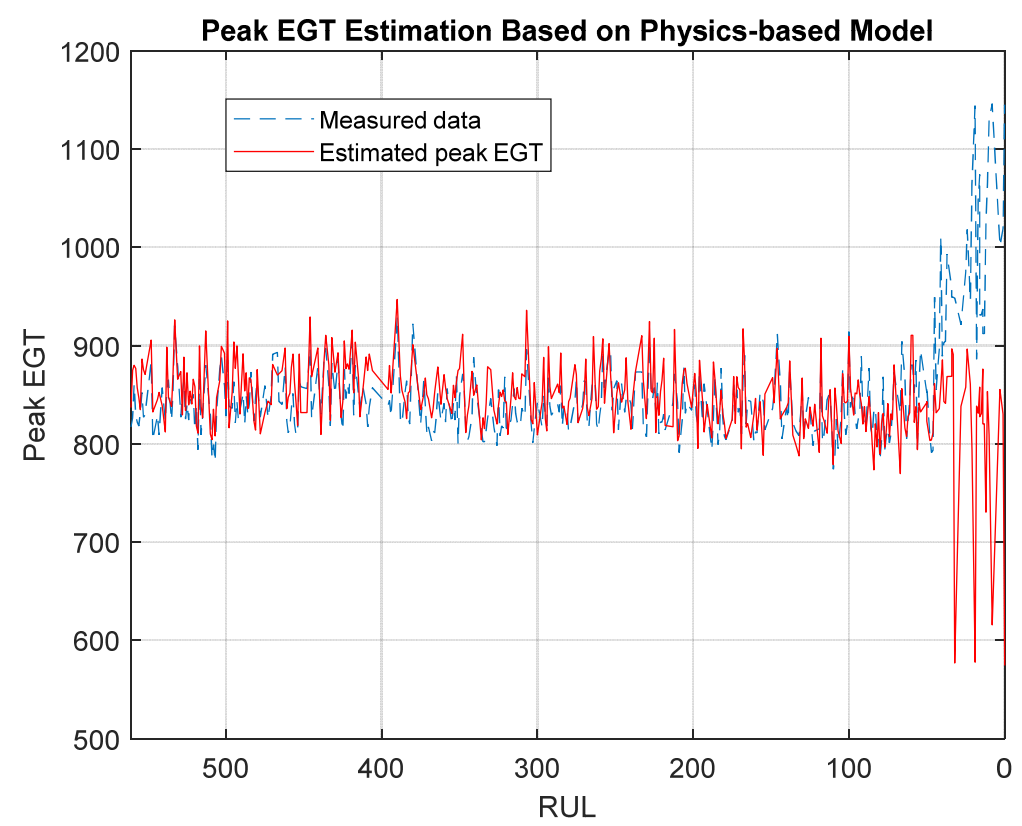

(b)

Figure 3.5 Implementation in faulty condition with physics-based model.

The neural network predicted $E G T_{\text {peak }}$ values are apparently higher than the corresponding values predicted by the physics-based models. The background knowledge on the gas turbine can be referenced to verify which model predicts the faulty condition more accurately. In the healthy condition, EGT gradually gets higher with increasing shaft speed before the $E G T_{\text {peak }}$ [62], while $N_{\text {peak }}$ always occurs at around $40 \%$ of the shaft speed at the design point $N_{\text {des }}$. When checking the shaft speed after RUL=125 in case (a) and RUL $=50$ in case (b), the data shows the $N_{\text {peak }}$ gradually decreases to $20 \%$ of $N_{\text {des }}$. Estimated EGT should get lower based on decreased shaft speed as presented in Fig. 3.5 rather than higher as suggested in Fig. 3.6, if the starter is in the healthy condition. Therefore, the physics-based transient model presents a more reasonable estimation for the starter faulty condition. 


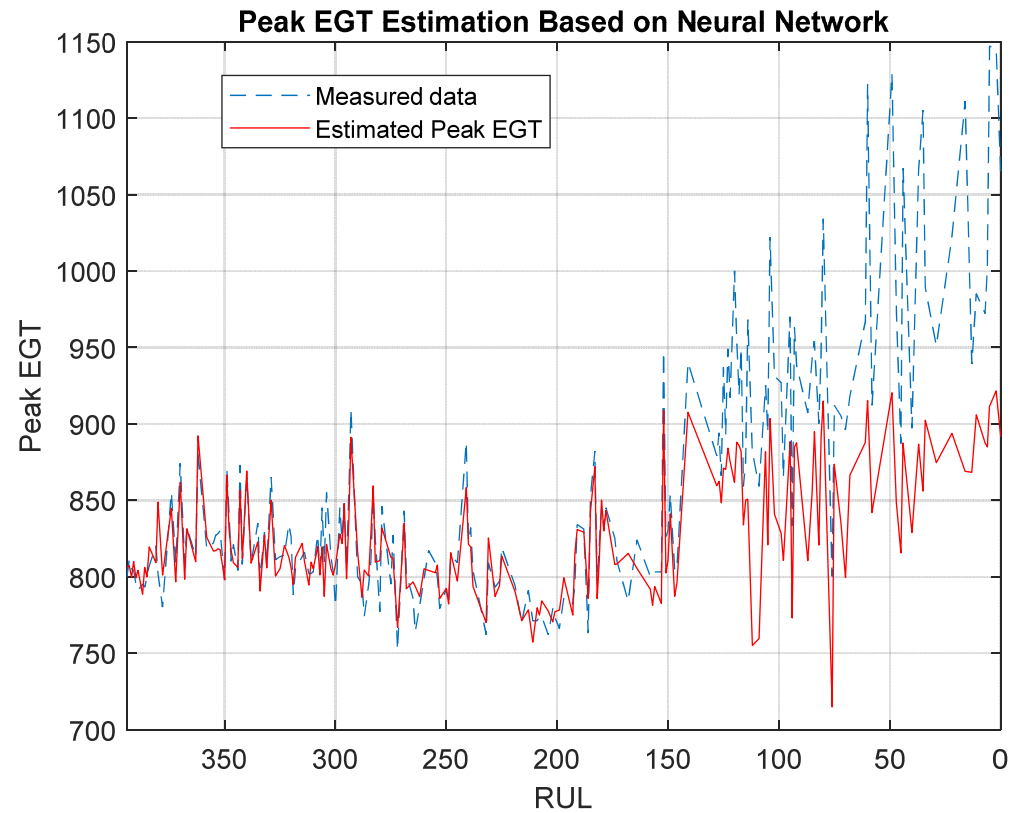

(a)

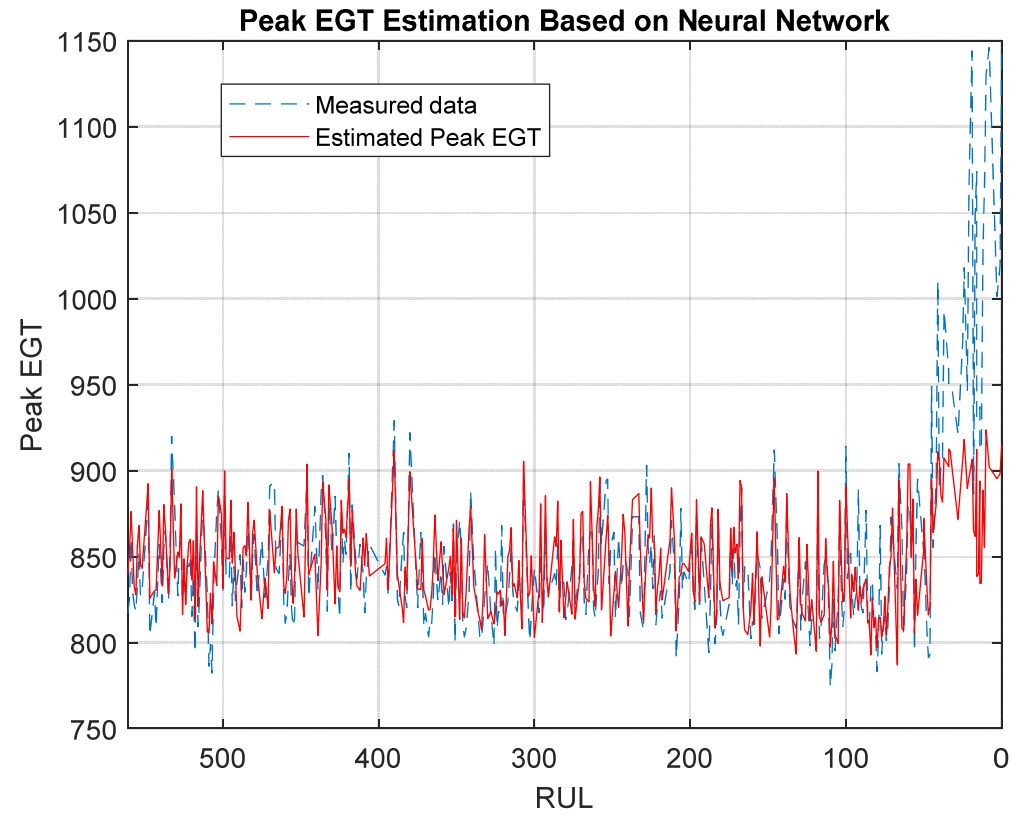

(b)

Figure 3.6 Implementation in faulty condition with neural network model. 


\subsection{Comparison of Two Models}

In starter healthy condition, both models can estimate $E G T_{\text {peak }}$ within acceptable accuracy while the neural network model demonstrates more accuracy compared to the physics-based approach. This can be explained with the fact that the training data within the neural network architecture cover the entire operating envelope in the healthy condition, and the corresponding interpolation provides a more accurate estimation accordingly.

In starter faulty condition, both models show a clear symptom through the deviation between the estimated and the measured values. However, the estimated results of the faulty starter by neural network are not reasonable at some condition, considering the background knowledge on the gas turbine performance. This highlights the limitation of the neural networks when applied for extrapolation.

Neural networks need less computational effort [77], [78] compared to thermodynamic modeling that involves a set of non-linear equations and a large number of iterations. Also neural networks show a prominent adaptive feature [78], involving the tune-up in the training process while a huge effort is required for adjusting the physics-based model for appropriate estimation. Nevertheless, due to the lack of analytical insight to the performance, neural networks are prone to misleading to erroneous results.

\subsection{Summary}

In this chapter, the neural network model is constructed for monitoring performance of the APU and comparing to the physics-based results. Both models are implemented in both the starter healthy and faulty conditions, and the results are analyzed and compared. 
Both methodologies show capabilities of estimating the starter performance and illustrating symptoms of failures, necessary for condition-based maintenance. Comparing the results from either methodology, the physics-based approach provides more accurate results on the starters with growing degradations. Whereas, neural networks perform more accurately with the starters at healthy condition. Fusion of both methodologies can therefore have a good potential for future work. 


\section{Chapter: Health Indicators for Starter Health Monitoring}

\subsection{Overview}

To quantitively predict the degradation severity and the health condition of the starter, two health indicators are proposed to identify the starter failure at the system level and component level respectively. The discrepancy between the measured Exhaust Gas Temperature $(E G T)$ and the corresponding predicted values from the model is proposed as the first indicator. The second indicator is defined as the ratio of the actual maximum power of the starter with respect to the healthy condition. It is specifically created for starter performance estimation and fault diagnostics at the component level, which only takes into account the health condition of the starter rather than other components of the APU. Both health indicators are derived from the developed physics-based model of the APU and have intuitive physical meanings.

\subsection{EGT Deviation Indicator}

Health indicators are assigned to quantify the symptoms of degradation for the components. A failure is declared when the indicator values exceed a designated threshold. The following health indicators are derived from the established physics-based model for the starter fault diagnosis.

Diagnostic approaches typically calculate the difference between the measurements and the model predicted values to quantify the degradation effects [76]. The closer the measured and the predicted values, the healthier the components are presumed. In this work, deviation of the EGT from the expected values based on the same shaft speed is 
introduced as a health indicator for the starter failure. When the starter gets degraded, its driving torque for the APU rotor acceleration diminishes and the rotor acceleration declines [86]. The control system of the APU reads the shaft speed and commands for injection of more fuel, presumably to compensate the delayed acceleration [74]. This leads to a higher $E G T_{\text {peak }}$, in case the starter is degraded. Meanwhile, the model predicts the expected $E G T_{\text {peak }}$ cal with smaller values, presuming its healthy performance condition. The difference of the measured and predicted EGTs reflects the degradation severity of the starter and is a suitable candidate for the starter health indicator.

$$
I_{E G T}=\left(E G T_{\text {peak }}-E G T_{\text {peak }_{c a l}}\right) / E G T_{\text {peak }}
$$

\subsection{Starter Health Indicator}

In this study, the performance of the starter in both healthy and faulty condition during the startup phase are schematically presented in Fig. 4.1.

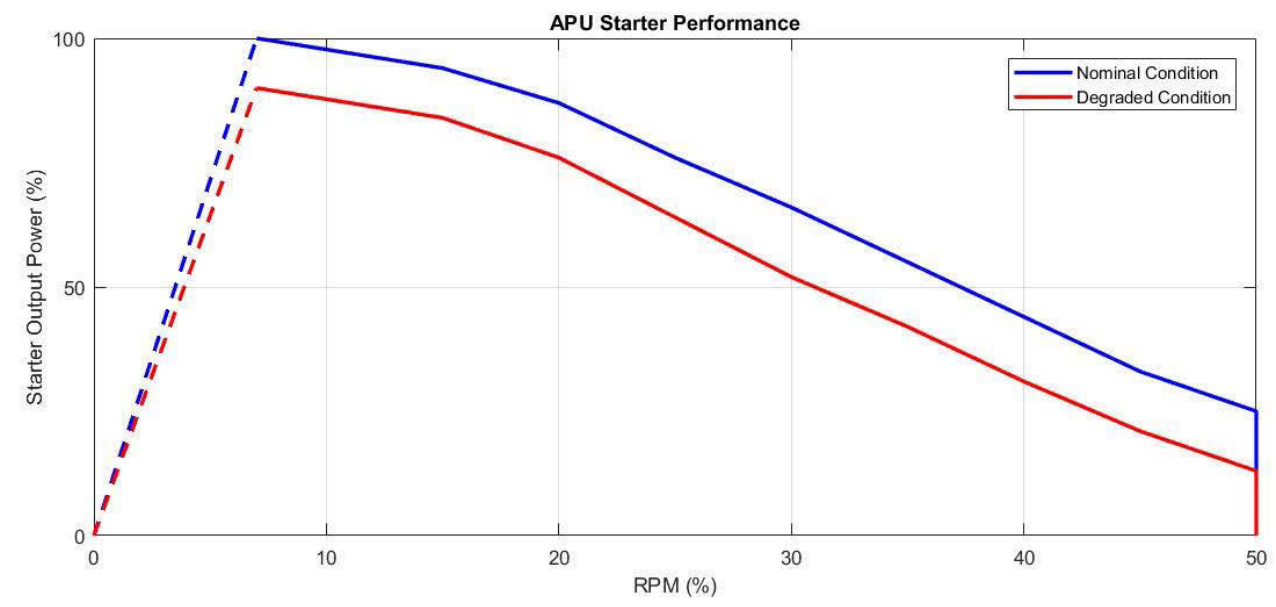

Figure 4.1 APU starter performance during the startup phase. 
As the interpretation in Chapter 2, the physics-based model for APU starting process is cited again.

$$
F\left(T_{a m b}, P_{a m b}, N_{\text {peak }}, \alpha, P W_{s}, E G T_{\text {peak }}\right)=0,
$$

As the starter power $P W_{s}$ and the rotor acceleration $\alpha$ in Eq. (4.2) are affected by the starter degradation, two health state parameters, starter health indicator and acceleration decline factor are introduced in the thermodynamic model of starting the APU. Starter health indicator $I_{S}$ is defined as the ratio of the starter maximum output power in a faulty condition and that in the healthy condition.

$$
I_{s}=P W_{s, \max }(\text { faulty condition }) / P W_{s, \max }(\text { healthy condition }) .
$$

And the acceleration decline factor $a_{d}$ is defined as the ratio of the rotor acceleration in a faulty condition and that in the healthy condition. Due to lack of data, the acceleration decline factor is assumed unchanged during a single starting cycle.

$$
a_{d}=\alpha(\text { faulty condition }) / \alpha(\text { healthy condition })
$$

Therefore, with Eqs. (4.2), (4.3) and (4.4), the thermodynamic model of starting the APU applied in the starter fault diagnosis is expressed as the following functional form.

$$
F\left(T_{a m b}, P_{a m b}, N_{\text {peak }}, E G T_{\text {peak }}, \alpha, a_{d}, P W_{s, \text { max }}, I_{s}\right)=0 .
$$


As the acceleration changes with the starter degradation, the starting time $(t)$ is affected. The time before the ignition (7\% shaft speed) is affected by $I_{s}$ while $a_{d}$ impacts the time between the ignition and the starter disengagement (50\% shaft speed) [1], [86].

Start time of the APU includes three stages as following:

$$
t=t_{1}+t_{2}+t_{3}
$$

Stage 1: APU shaft speed is under $7 \%$, and only the starter provides power to accelerate the rotor. Therefore, $t_{1}$ is influenced only by the starter health indicator.

$$
t_{1}=t_{1}\left(I_{s}\right)
$$

Stage 2: APU shaft speed is between $7 \%$ to $50 \%$, and both the starter and the turbine provide power to accelerate the rotor. $t_{2}$ is a function of the acceleration decline factor.

$$
t_{2}=\int_{7 \% N}^{50 \% N} \frac{1}{\alpha(N) \times a_{d}} d N
$$

Stage 3: APU shaft speed is above $50 \%$. At $50 \%$ of shaft speed, the starter disconnects from the APU shaft. In this condition, the power is maintained only by the APU turbine section. Degradation of the starter has no effect on $t_{3}$, since there is no starter assistant power during this Stage. As a result, $t_{3}$ remains unchanged while the starter gets degraded. 
From Eqs. (4.6) through (4.8), starting time is found as:

$$
t=F\left(I_{s}, a_{d}\right)
$$

With Eqs. (4.5) and (4.9), only two parameters, starter health indicator and acceleration decline factor are unknown. Therefore, the starter health indicator can be defined as:

$$
I_{s}=F\left(T_{a m b}, P_{a m b}, N_{\text {peak }}, E G T_{\text {peak }}, \alpha, a_{d}, P W_{s, \text { max }}, t\right)
$$

\subsection{Implementation Results for EGT Deviation Indicator}

In each starting cycle, EGT deviation indicator and starter health indicator are derived with Eqs. (4.1) and (4.10) respectively. To reveal the long-term trends of the indicators, a moving average data-smoothing filter is used to remove the momentary fluctuations.

In healthy condition of the starter, the EGT deviation indicator does not vary much during the whole operating life, and fluctuates around zero as shown in Fig. 4.2. This implies the starter has stayed in healthy condition during the period data collected. The moving average spanned over 20 time steps is shown in red solid line in the plots to display the underlying trend.

In starter faulty condition, a different trend shows up in Fig. 4.3. At the beginning, the EGT deviation indicator maintains around zero. After around RUL=125 in case (a) and $\mathrm{RUL}=100$ in case (b) respectively, the $E G T$ deviation indicator grows rapidly. The closer 
it gets to the final failure, the faster it increases. That implies the starter is degrading more and more, and eventually, it is unable to start the APU.

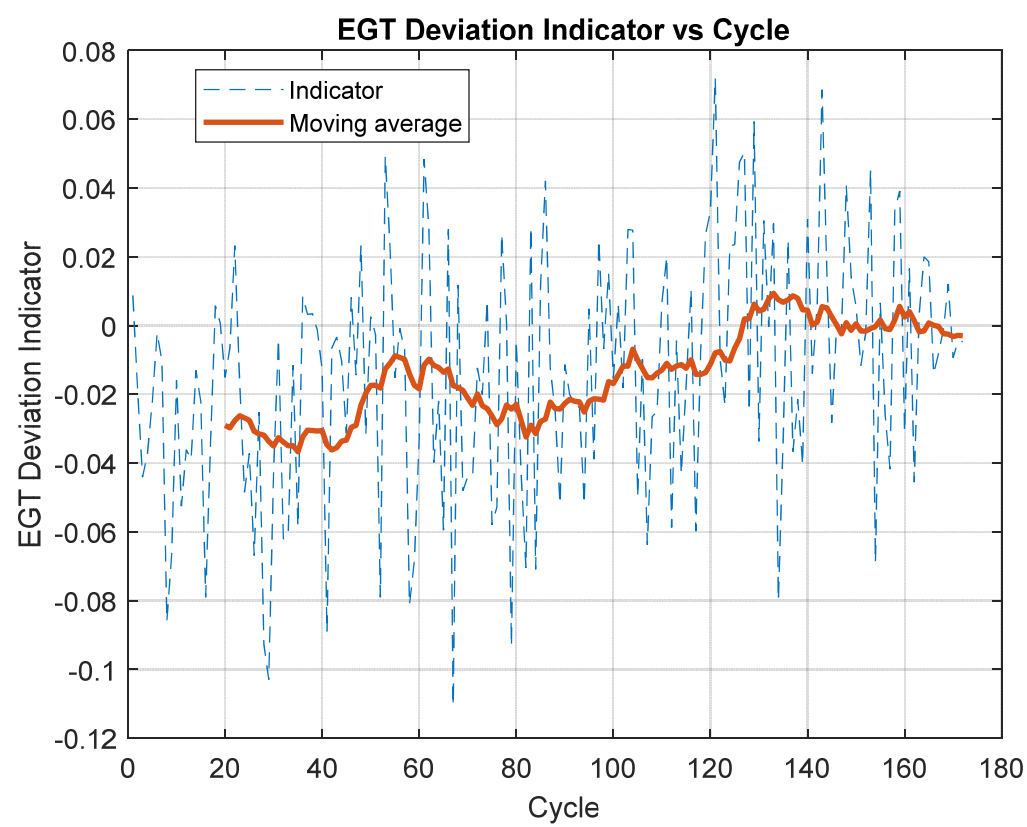

(a)

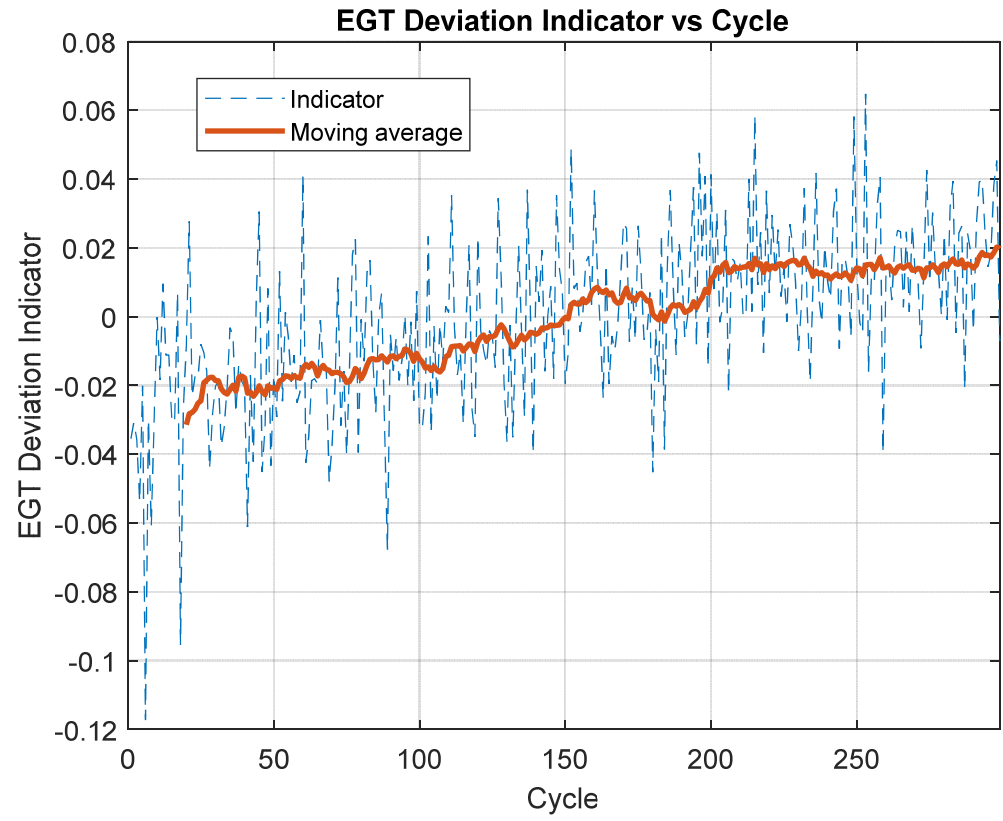

(b)

Figure 4.2 $E G T$ deviation indicator in starter healthy condition 


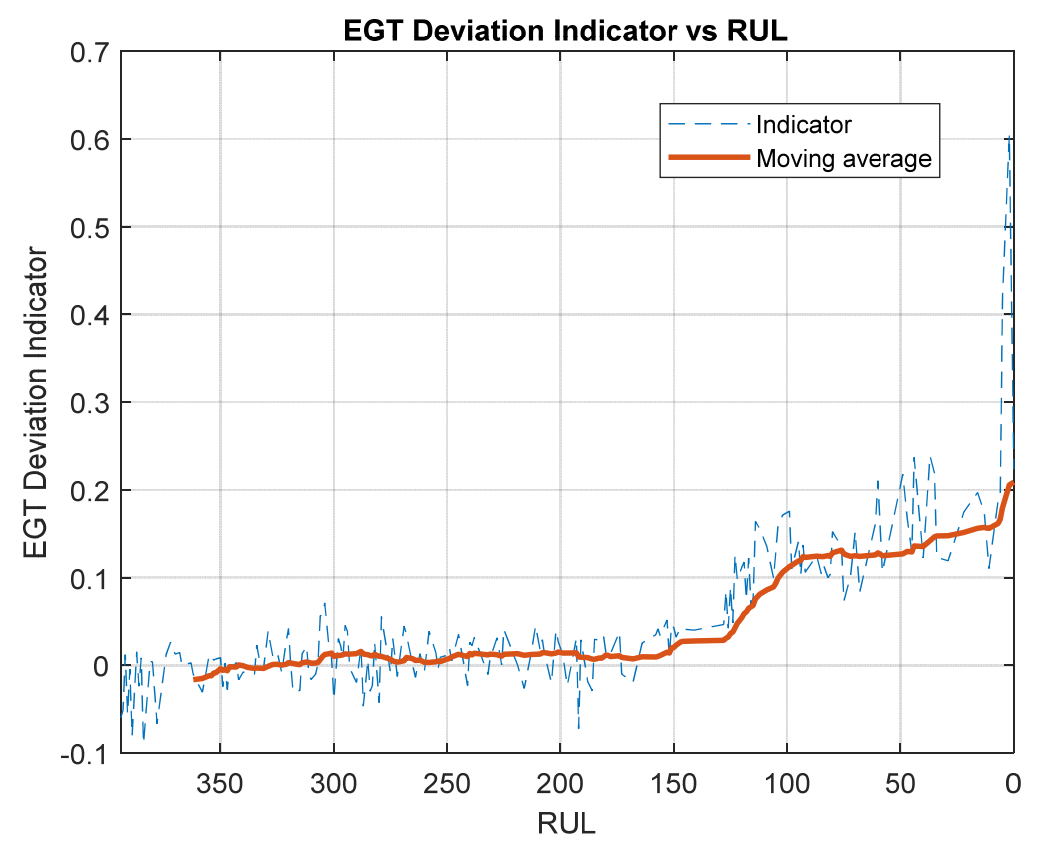

(a)

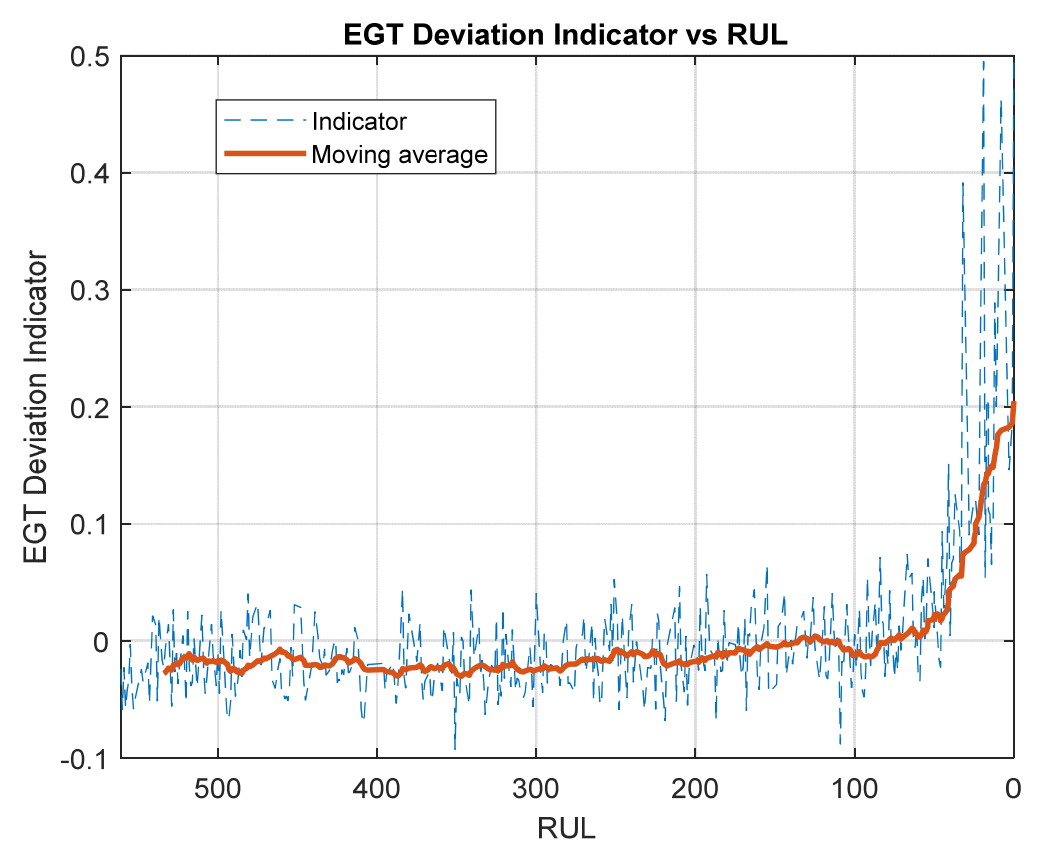

(b)

Figure 4.3 EGT deviation indicator in starter faulty condition 


\subsection{Implementation Results for Starter Health Indicator}

Figures 4.4 and 4.5 present variations of the starter health indicator for APUs in healthy condition and those with a faulty starter. In healthy condition, the starter health indicator varies around unity, as shown in Fig. 4.4. That implies the output power of the starter has not tangibly changed with respect to the initial power level of the new condition.

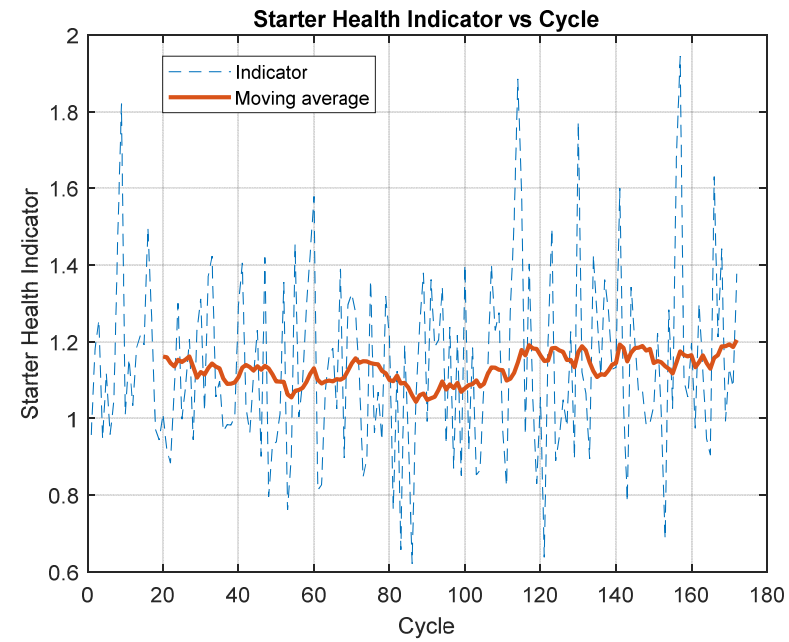

(a)

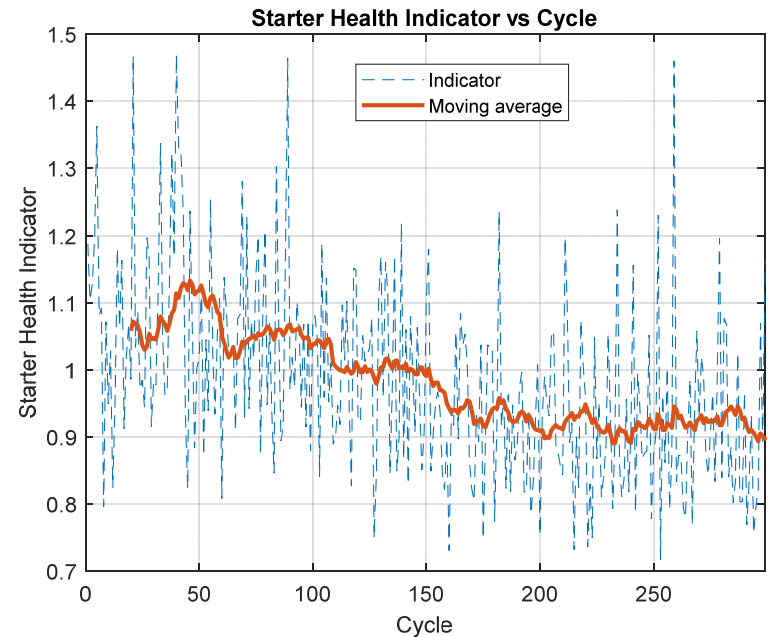

(b)

Figure 4.4 Starter health indicator in starter healthy condition 


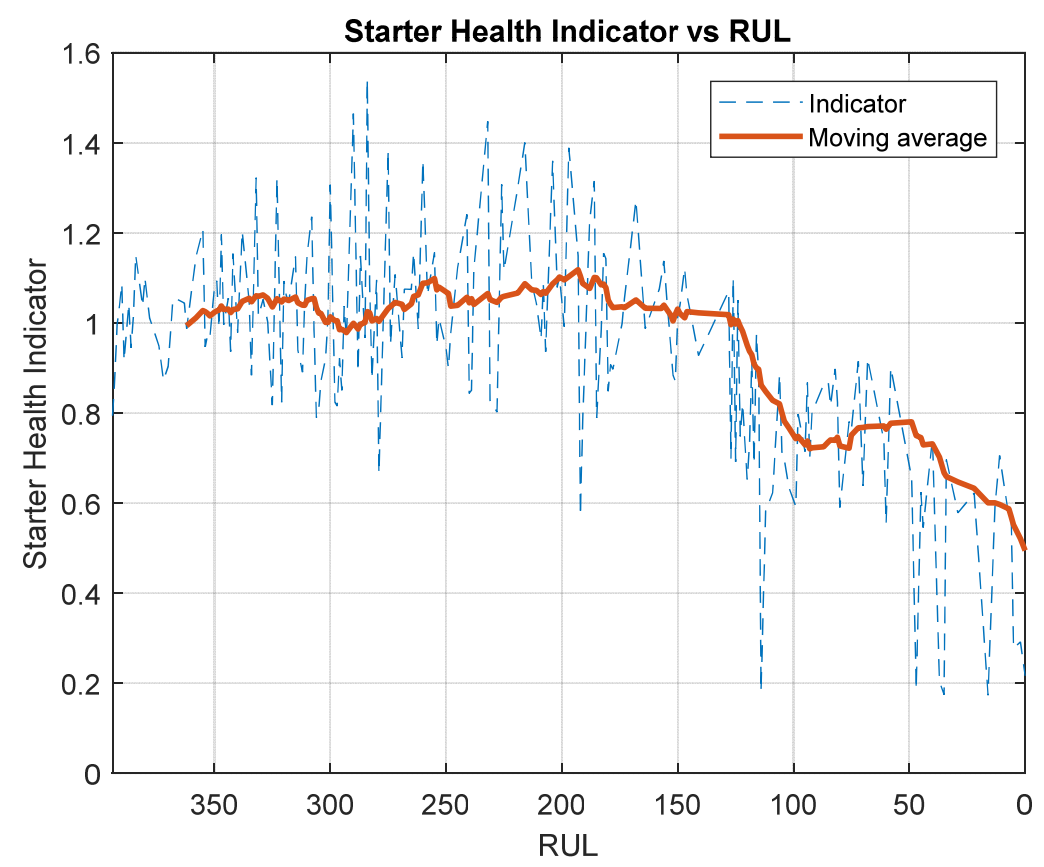

(a)

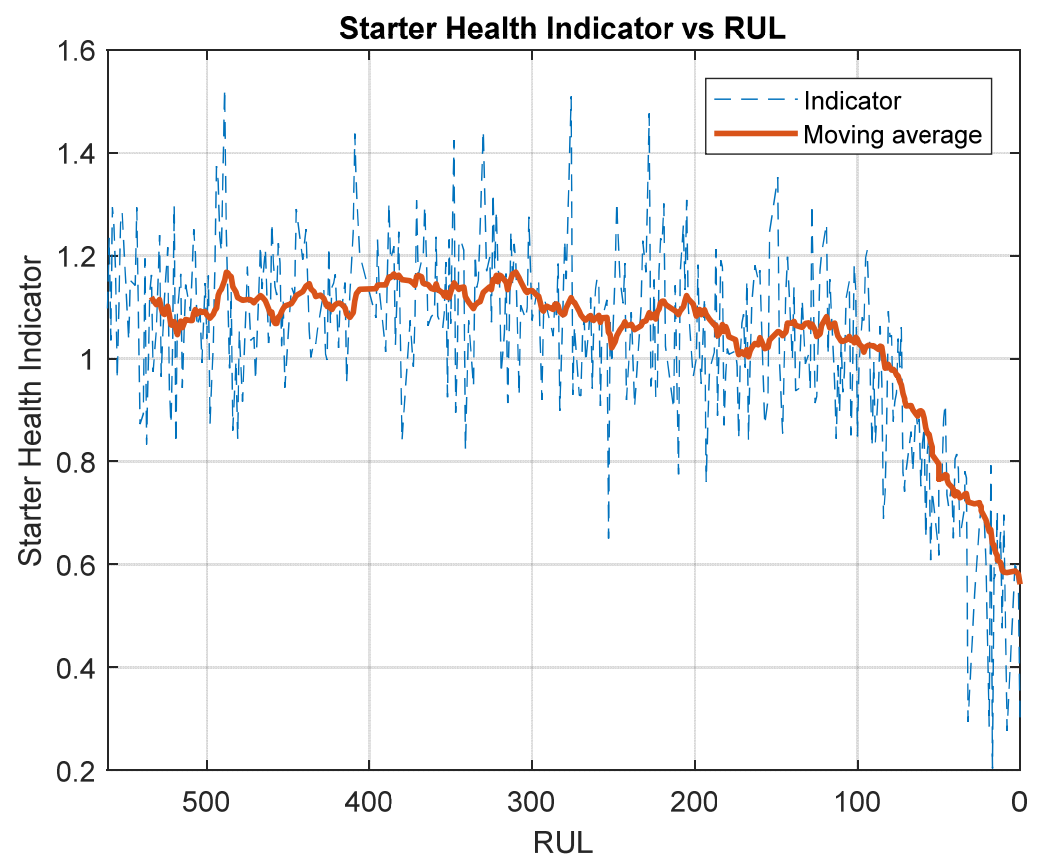

(b)

Figure 4.5 Starter health indicator in starter faulty condition 
A different pattern is exhibited in Fig. 4.5. At the beginning, the indicator has remained close to unity, and then it begins to decline approximately at RUL $=125$ in case (a) and RUL=100 in case (b) respectively. The failure has happened when the indicator value has fallen to around 0.5 .

\subsection{Comparison of Two Indicators}

The results of the two indicators comply with each other. They both present a steady trend in starter healthy condition and large variations in starter faulty condition. Both indicators demonstrate effectiveness to quantify the degradation level of the starter and provide condition-based information on the health state of the starter, required for CBM.

Compared to the EGT deviation indicator, the starter health indicator is restricted to the effects of the starter faults whereas the EGT deviation indicator could potentially be affected by degradation of the other components of the APU. Therefore, the starter health indicator is potentially a better candidate for maintenance personnel to identify the starter health condition.

\subsection{Summary}

To improve performance monitoring and malfunction prediction of starters, two health indicators are developed based on the physics-based model of the APU starting process. Both indicators quantify the health condition of the starter and provide diagnostic information with no need to have the past measurements from the system. The EGT deviation indicator presents the deviation between the predicted EGT values and the measured ones, whereas the starter health indicator provides the ratio of the maximum 
output power in faulty condition compared to that in healthy condition. The results with both indicators clearly show the degradation severity for the cases with faulty starters. Both indicators hold explicit physical meanings. With limited measurements from the available sensors in an aircraft, the developed technique and the indicators can provide monitoring tools and measures of reliability for the starter system of the APU. 


\section{Chapter: Starter Fault Diagnostics}

\subsection{Implementation of Individual Indicators for Diagnosis}

Diagnostics includes fault detection and isolation. Performance-based diagnostics is a powerful diagnostic approach widely used in the gas turbine industries [76]-[78], [87]. This approach attempts to detect and monitor degradation of the components through their effects reflected in deterioration of performance parameters measurement. Commonly used performance parameters include gas-path temperatures and pressures, rotational speed, fuel flow and power [9]. Features are extracted from the performance parameters to be used for diagnosis. The features are developed mainly using physicsbased models and/or data-driven techniques. The indicators developed in Chapter 4 are based on physics-based models.

To classify the state of the starter, a threshold should be assigned as a boundary between the healthy and faulty states. The threshold is set as a value, at which the failure indication is triggered once the value of the indicator is exceeded.

The dataset available in this work is collected from the flight records of a fleet of commercial aircraft, and includes 11 cases with starter faulty condition and 14 cases with starter healthy condition. The life cycle of the starter is up to 8000 starts based on the product specifications provided by the manufacturer. To populate failure data, the last 10 start records leading to the final failure are labeled as faulty that amount to 110 cases in total.

The non-parametric kernel density estimation (KDE) is used to estimate the continuous probability density function (PDF). Unlike parametric distribution, kernel 
distribution does not assume the distribution of the data, thereby it avoids introducing unforeseen uncertainties [88]. KDE is defined as

$$
\hat{f}(x)=\frac{1}{n b} \sum_{i=1}^{n} K E\left(\frac{x-x_{i}}{b}\right)
$$

where, $x_{i}$ is a random sample from an unknown distribution, $n$ is the sample size, $K E(\cdot)$ is the kernel smoothing function, and $b$ is the bandwidth. KDE is characterized by the smoothing function $K E(\cdot)$ and the bandwidth $b$, which determine the smoothing results of the curve. In this study, Gaussian distribution is assumed in the smoothing function and $b$ is optimized by Silverman's rule of thumb [89].

Using the failure data, a histogram is established with respect to the EGT deviation indicator, by a moving average, as shown in Fig. 5.1. (a). The histogram exhibits the shape of the underlying density distribution and PDF is estimated using KDE, shown in red in the same plot. Figure 5.1. (b) shows cumulative distribution function (CDF) of failures with respect to the $E G T$ deviation indicator and a threshold at $90 \%$ fault detection probability is shown as an example. That means $90 \%$ of failures are detected by setting the threshold at this value. The fault detection probability is defined as follows.

$$
\text { Fault detection probability }=\frac{\text { detected failures }}{\text { Total failures }}
$$

With the same approach, the threshold for the starter health indicator is determined across the entire detecting range, shown in Fig. 5.2 


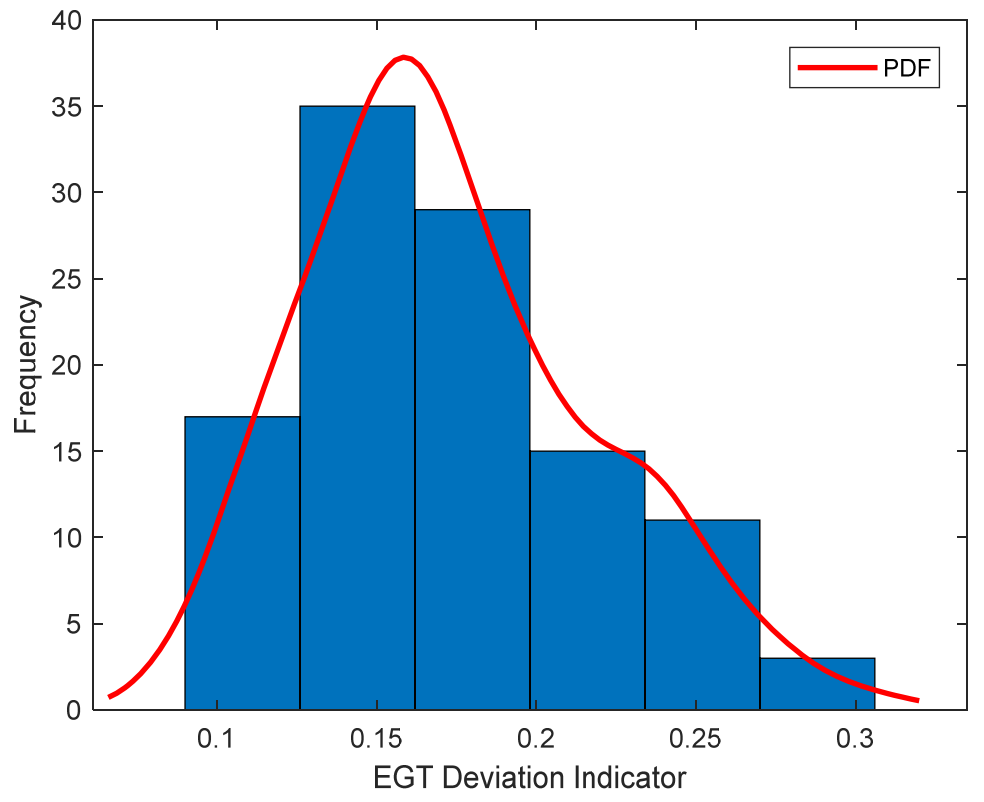

(a)

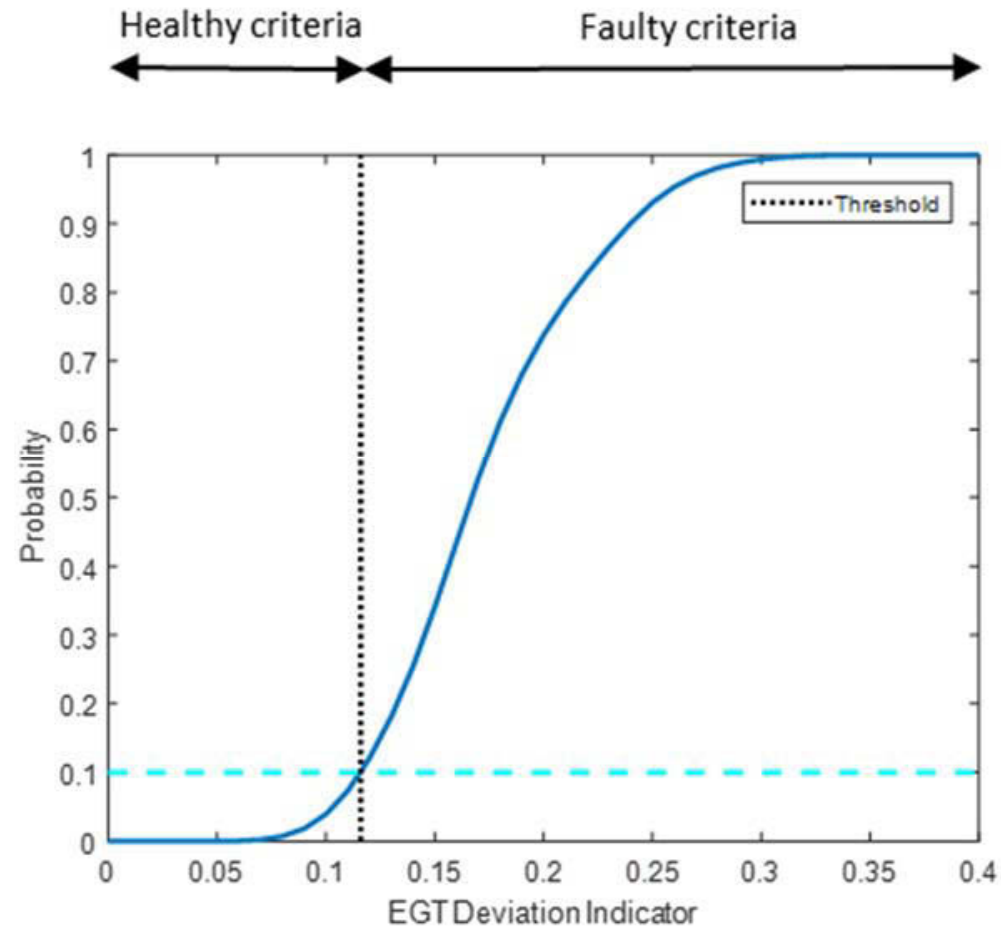

(b)

Figure 5.1 PDF and CDF of failures with respect to the $E G T$ deviation indicator 


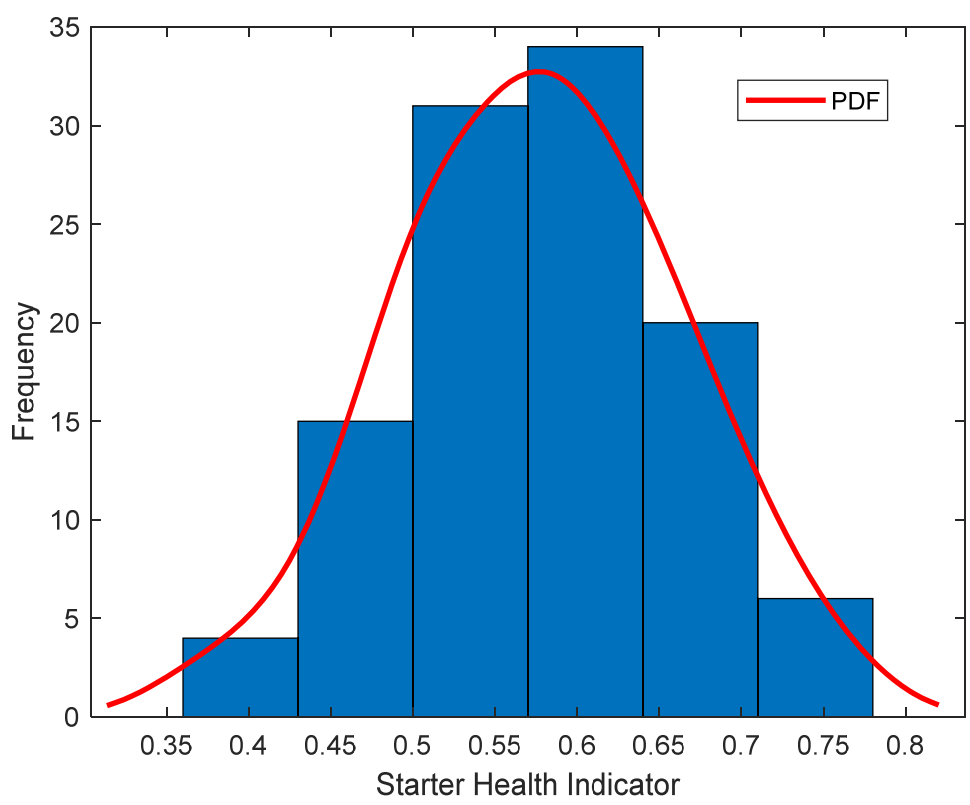

(a)

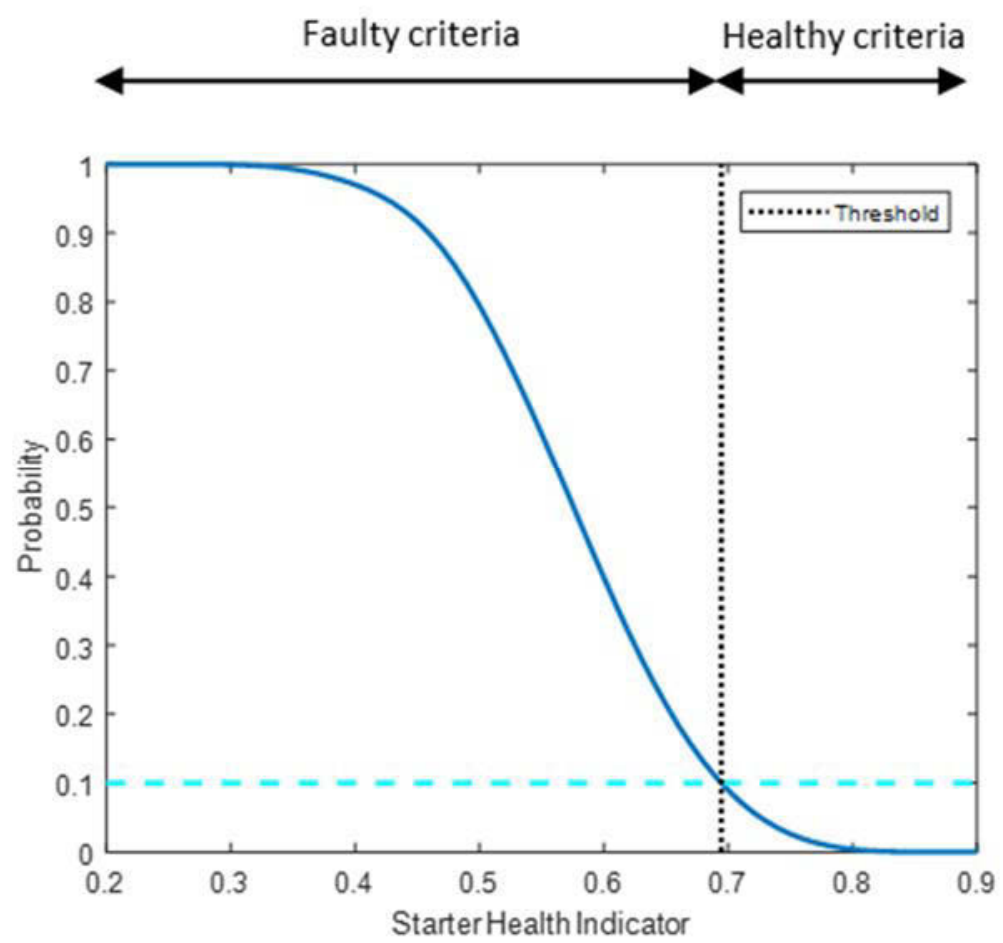

(b)

Figure 5.2 PDF and CDF of failures with respect to the starter health indicator 
By this stage, the thresholds for both individual indicators are defined. The typical implementation of individual indicators for diagnosis are shown in both Fig. 5.3. and Fig.

\section{4 .}

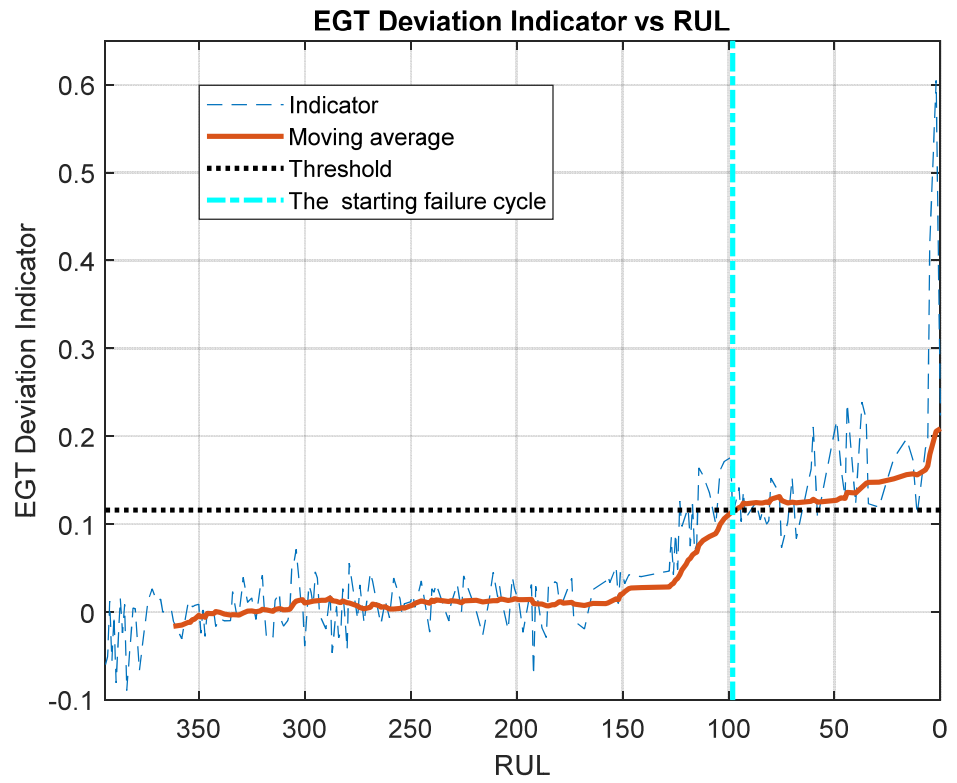

(a)

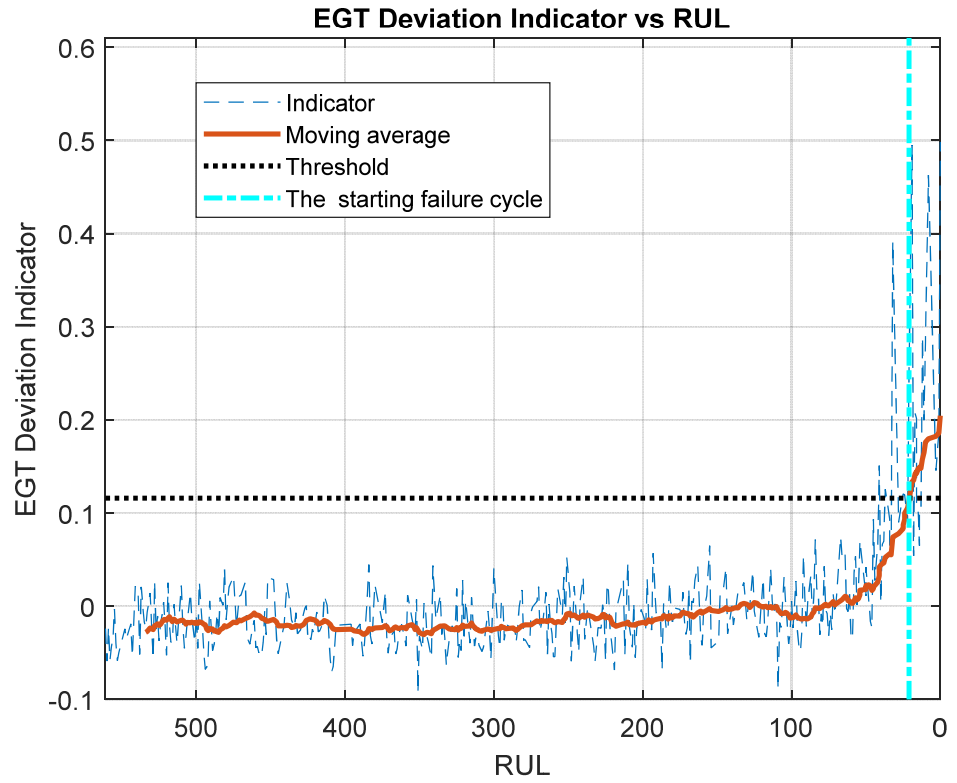

(b)

Figure 5.3 Implementation of the $E G T$ deviation indicator for diagnosis 


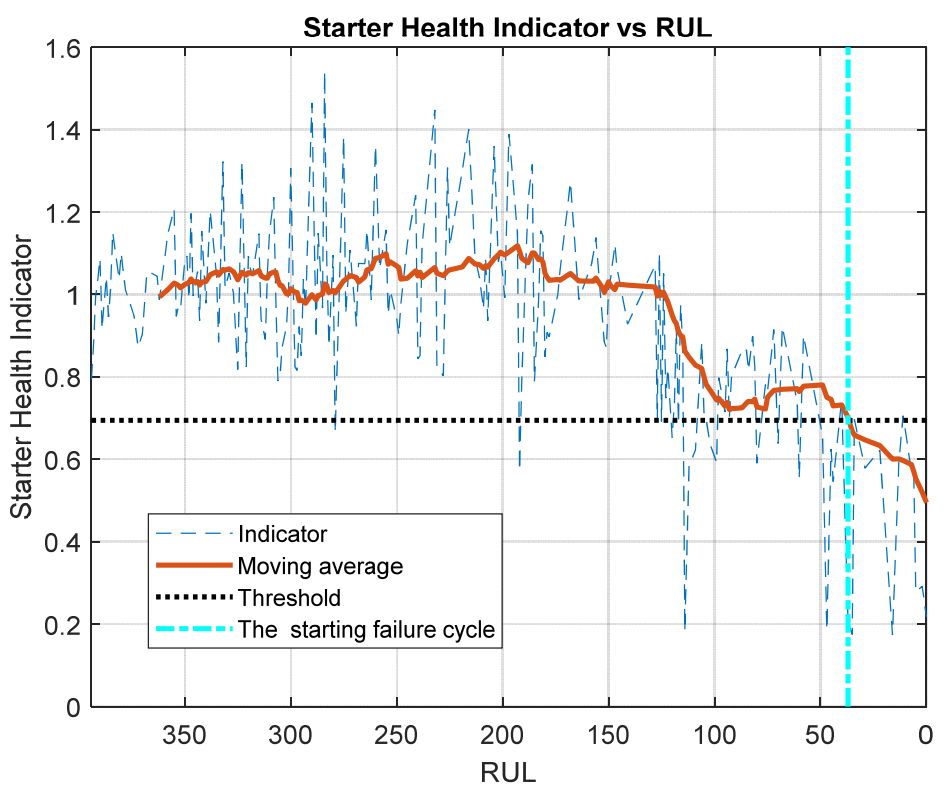

(a)

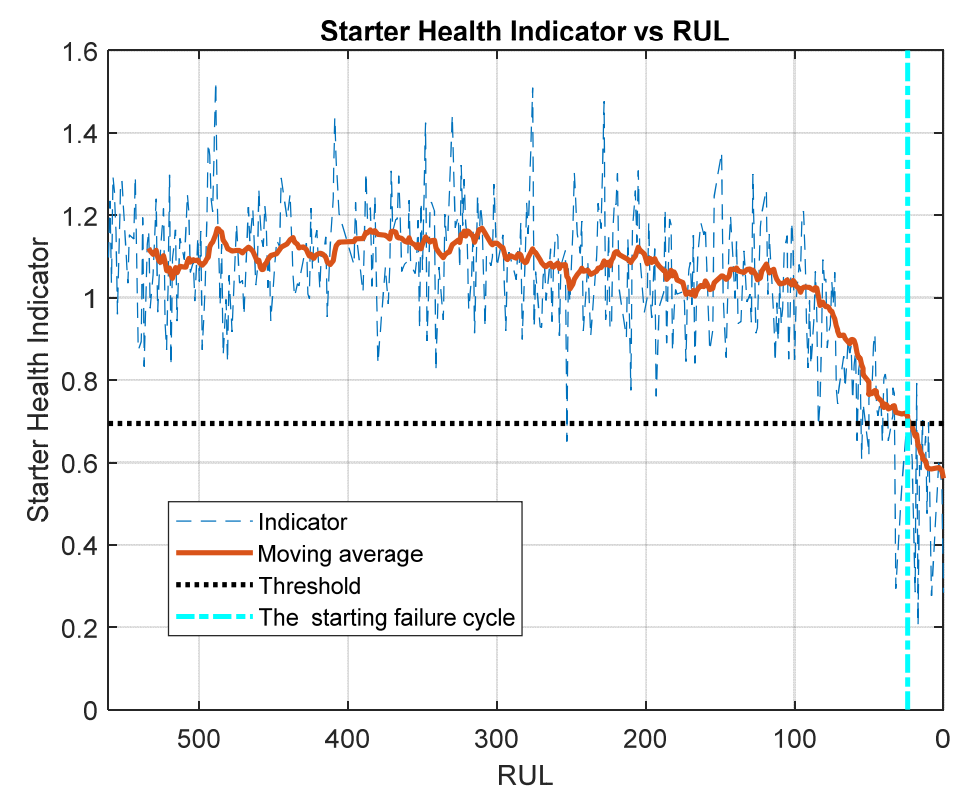

(b)

Figure 5.4 Implementation of the starter health indicator for diagnosis

The failure is declared when the indicator exceeds the threshold. The corresponding starting failure cycle is highlighted by vertical dash-dotted lines in cyan color. The approach provides significant information about the current health state and the expected 
time of the failure. As a result, the necessary maintenance action can be anticipated to restore the starter functionality. The threshold at $90 \%$ fault detection probability in this section is provided as an example. The approach to determine the optimal threshold is detailed in Chapter 6.

\subsection{Implementation of An Enhanced Joint Indicator for Diagnosis}

Traditionally, the threshold is determined in one dimension for individual indicators [57], [58]. That can cause confusion in the decision making when working with multiple indicators, as any indicator may exceed its respective threshold at a different time. With multiple indicators, if a failure is declared only when all thresholds have been exceeded, many failures cannot be detected, and the reliability of the diagnostic system would decline. To address this problem, a novel approach is proposed in this work to aggregate the indicators for simultaneous diagnosis in a multi-dimensional feature space. The joint probability distribution is used to develop the joint indicator, where joint PDF and the corresponding joint CDF is adopted to generate thresholds in the two-dimensional space.

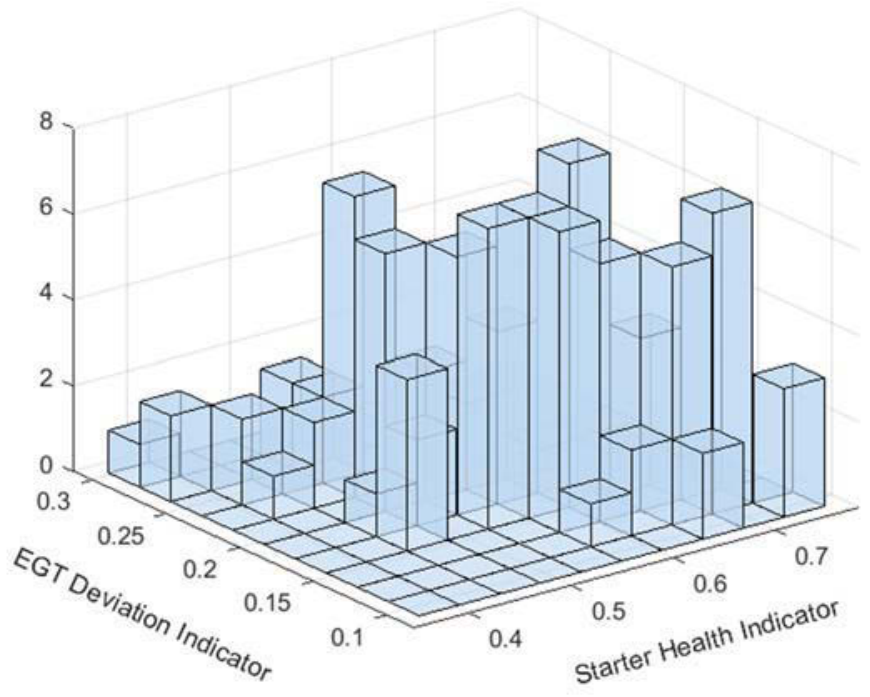

Figure 5.5 Bivariate histogram for both indicators 
Figure 5.5 illustrates a bivariate histogram associated with the data from both the EGT deviation indicator and starter health indicator.

To construct a continuous bivariate distribution, bivariate KDE is utilized, where the joint PDF is defined as,

$$
\hat{f}(x ; \mathrm{H})=\frac{1}{n} \sum_{i=1}^{n} K E_{H}\left(x-X_{i}\right)
$$

where, $X_{i}$ is a random sample from a bivariate distribution, $\mathrm{H}$ is the bandwidth matrix that is defined as a symmetric positive definite matrix, and the kernel smoothing function $K E(\cdot)$ used here is the standard bivariate normal density function.

$$
K E(x)=\frac{1}{2 \pi} \exp \left(-\frac{1}{2} x^{T} x\right)
$$

With applying two-dimensional KDE, the continuous joint PDF of the joint indicator is obtained, as shown in Fig. 5.6. The probability distribution of the joint indicator is defined by the volume under the density function. Figure 5.6 also shows the contours of the joint PDF where the red circles present starter incidents.

Figure 5.7. illustrates the joint $\mathrm{CDF}$ and the corresponding contours for the joint indicator. The CDF contours are instrumental to define the threshold for the joint indicator at different fault detection probability, e.g., when the threshold is assigned at the curve with 0.1 value in the plot, $90 \%$ of failures can be detected. The thresholds assigned 
in two-dimensional space apply both indicators values at the same time to diagnose the starter failures.
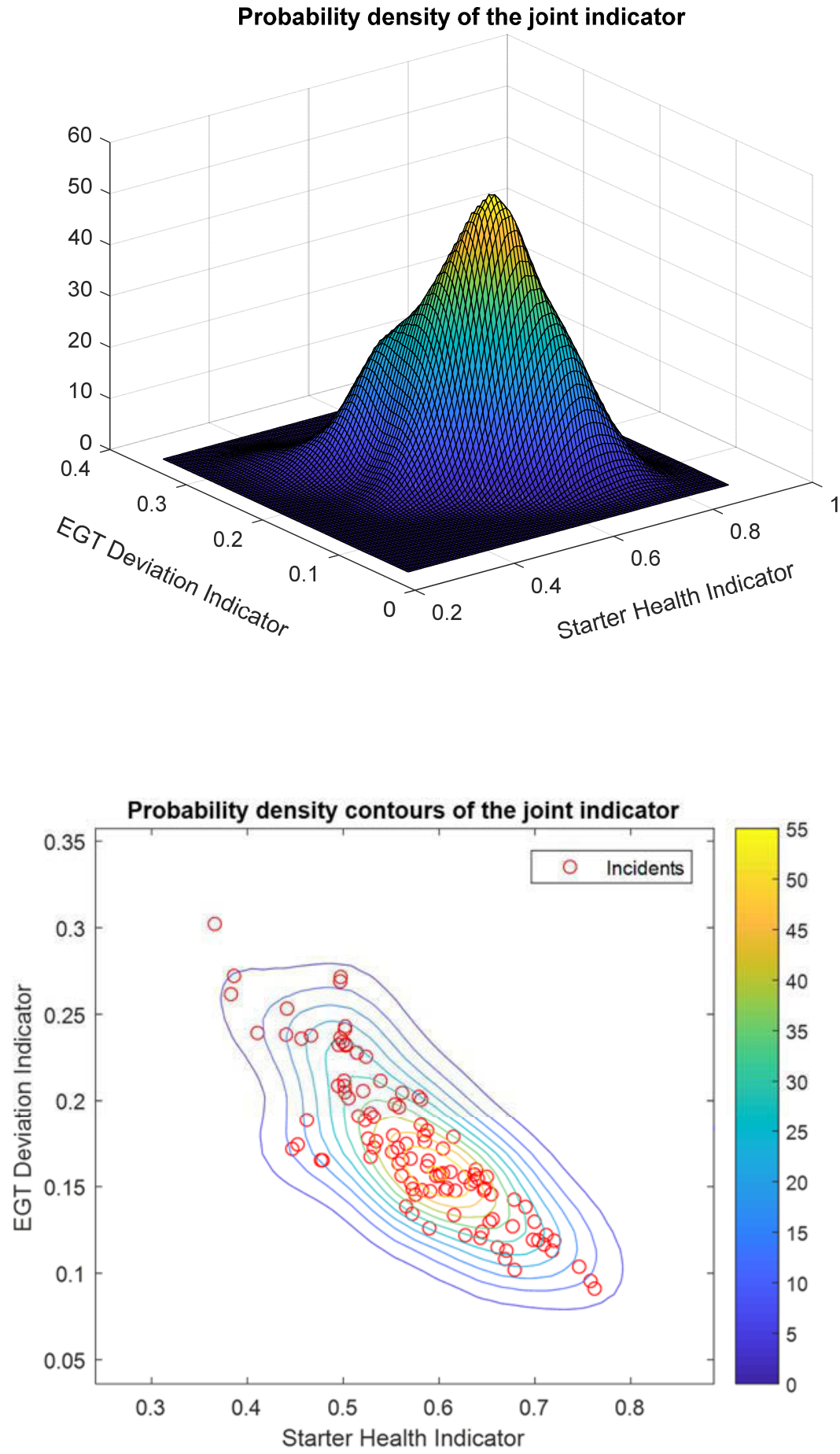

Figure 5.6 Joint PDF of the joint indicator 

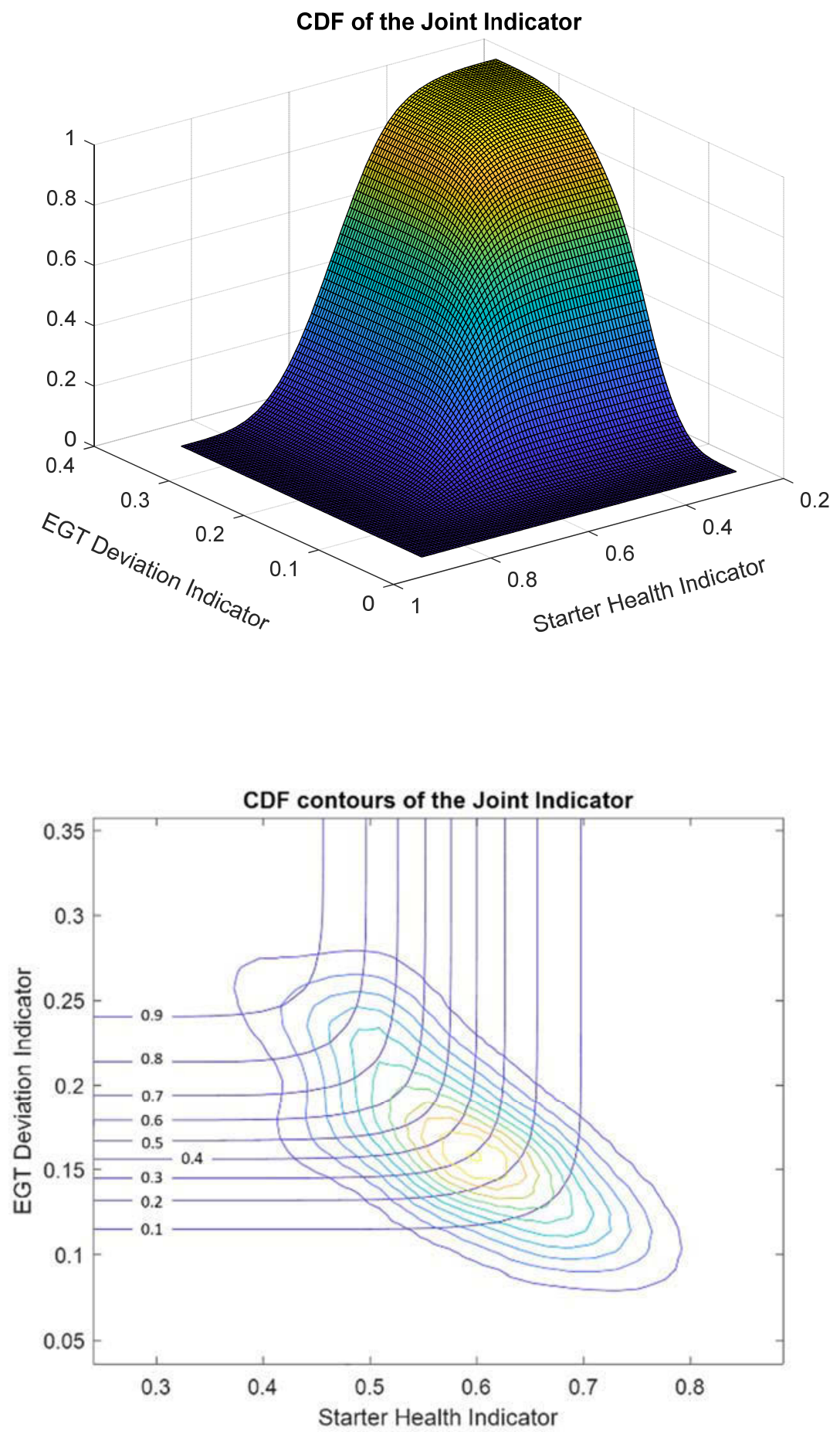

Figure 5.7 Joint CDF of the joint indicator

A typical implementation of the joint indicator is illustrated in Fig. 5.8. In the starter healthy condition, the indicator remains in the right bottom corner of the plot. With the 
degradation of the starter, the indicator gradually moves to the left top corner. The more the indicator approaches to the threshold, the more the starter approaches to the end of its life. A failure is declared as the indicator passes through the threshold. The predominant performance of the enhanced joint indicator and the detailed comparison with the two individual indicators are presented in Chapter 6.

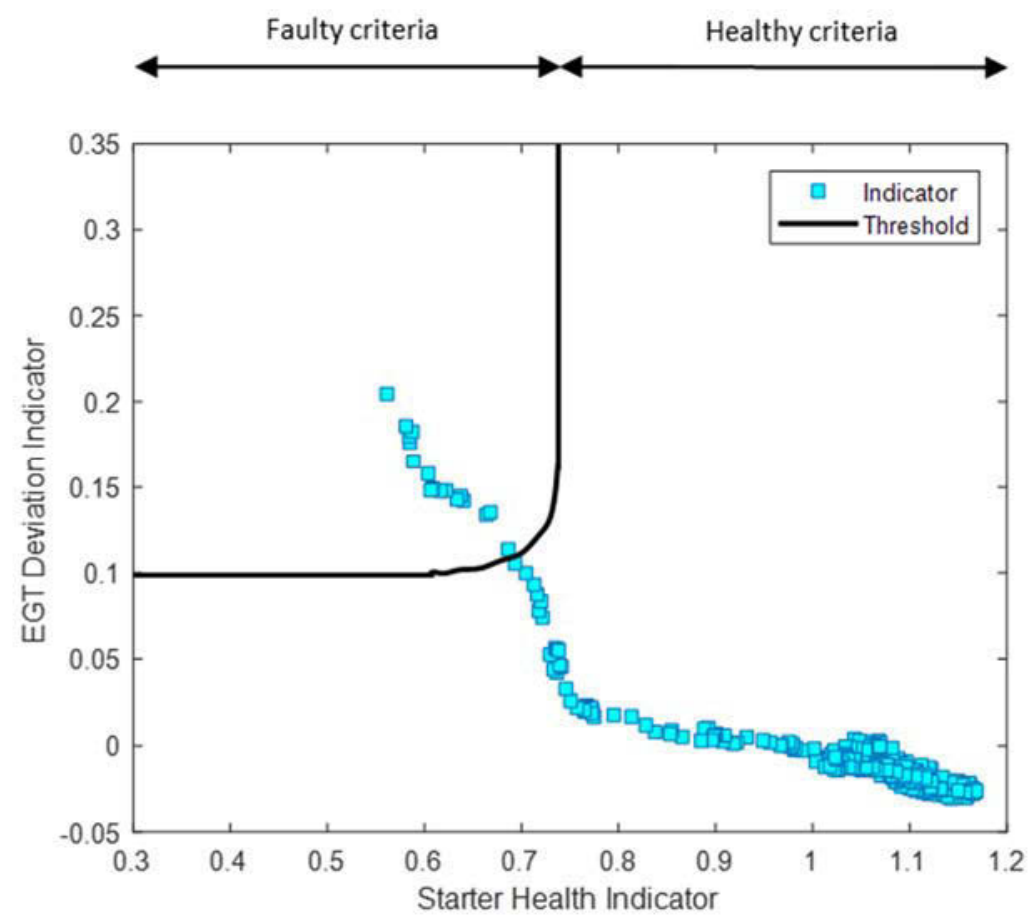

Figure 5.8 Implementation of the joint indicator for diagnosis

\subsection{Summary}

The implementation of individual indicators for diagnosis is firstly exhibited in this chapter. The threshold is defined in one dimension and the corresponding starting failure cycle is highlighted. To improve diagnostic performance, an enhanced joint indicator is developed based on the joint probability distribution of the two indicators and a non- 
linear threshold is assigned for classification of the healthy and the faulty conditions. The enhanced joint indicator aggregates the two individual indicators for simultaneous diagnosis in a multi-dimensional feature space and enables multiple-feature diagnosis for the starter. 


\section{Chapter: Evaluation of Diagnostic Results for Indicators}

\subsection{Receiver Operating Characteristic Curves}

A Receiver Operating Characteristic (ROC) curve is a graphical representation to visualize the diagnostic performance of indicators and facilitate comparison between distinct indicators. It provides important information, such as failure probability, diagnostic accuracy and the operating costs. These are all essential for the indicator evaluation and decision making based on the corresponding diagnostic performance. Due to its outstanding capability of evaluating the diagnostic performance of indicators, ROC curves are widely used in various industries [90]-[93]. The confusion matrix of ROC,

presented in Table. 6.1, explains the definition of the commonly used terminology in ROC.

Table 6.1 A confusion matrix of ROC

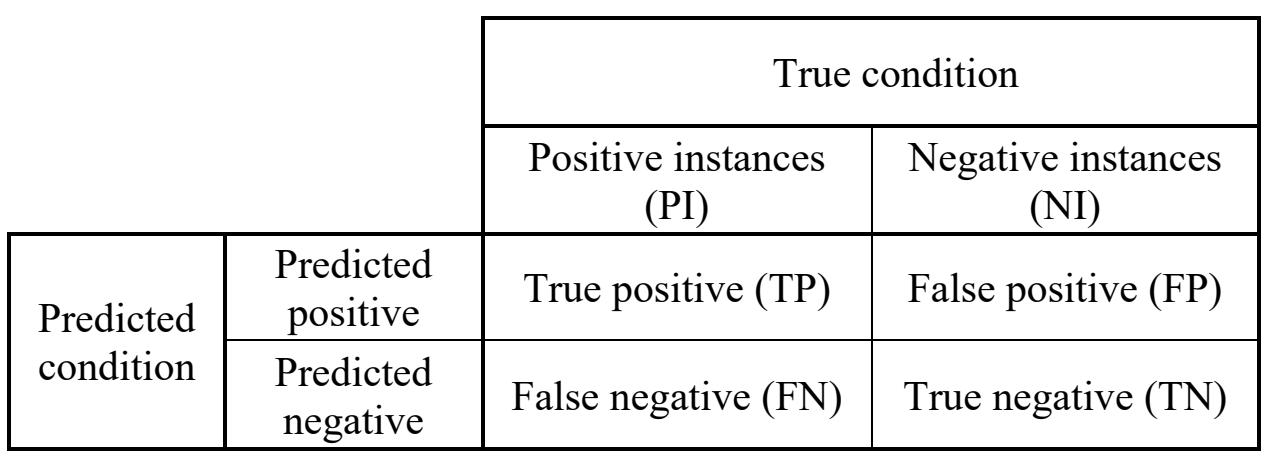

The ROC works for both continuous random variables and discrete random variables. It is generated by plotting the True Positive Rate (TPR) versus the False Positive Rate (FPR) with varying thresholds through the entire detecting range. TPR is defined as the ratio of true positive (TP) with respect to total positive instance (PI) whereas FPR is 
defined as the ratio of false positive (FP) and total negative instances (NI). Thresholds in this study are determined at various fault detection probability settings, from $0 \%$ to $99 \%$.

$$
\begin{aligned}
& T P R=T P / P I, \\
& F P R=F P / N I .
\end{aligned}
$$

TPR is also known as sensitivity while FPR is also called as probability of false alarm [94]. The ROC compares the mentioned two ratios to evaluate the diagnostic performance of indicators. A good indicator presents high sensitivity while reducing the false alarm ratio.

Another diagnostic metric is the diagnostic accuracy (ACC) that is defined as

$$
A C C=\frac{T P+T N}{P I+N I}
$$

\subsection{Optimal Threshold Determination}

Conventional diagnostics approaches defined the threshold of the indicator at a fixed number of fault detection probability, like $95 \%$. That is not usually the optimal threshold considering the specific case. One of the advantages to apply the ROC curve for evaluation is its capability to determine the optimal threshold for the indicator. With cost information, the ROC depicts the trade-off between the benefits and costs. The benefit means improving detection reliability and safety while the costs imply reducing operating expenses. A balance between the impacts of the two factors should be determined to 
provide necessary guidance in the diagnostic decision making. The premise to use this tool is that the cost information should be available in advance.

\subsection{Cost Considerations}

The expected cost of indicators depends on the individual cost components associated with four detection results in Table. 6.1 and the corresponding occurrence probability [64], [91].

$\operatorname{Cost}=\frac{T P \times \operatorname{Cost}(P \mid P)+F N \times \operatorname{Cost}(N \mid P)+F P \times \operatorname{Cost}(P \mid N)+T N \times \operatorname{Cost}(N \mid N)}{P+N}$

where $\operatorname{Cost}(N \mid P)$ represents the cost of misclassifying a positive instance as a negative condition, $\operatorname{Cost}(P \mid N)$ is the cost of misclassifying a negative instance as a positive condition, and $\operatorname{Cost}(P \mid P)$ and $\operatorname{Cost}(N \mid N)$ are defined in a similar way. Equation (6.3) can be seen as a straight line with $F P R$ as the horizonal axis and $T P R$ as the vertical axis, as shown by the red dashed line in Fig. 6.1. The slope of the straight line $S$ is defined by,

$$
S=\frac{\operatorname{Cost}(P \mid N)-\operatorname{Cost}(N \mid N)}{\operatorname{Cost}(N \mid P)-\operatorname{Cost}(P \mid P)} \times \frac{N}{P}
$$

This straight line moves from the upper left corner within the ROC plot, point $[0,1]$ down to the right until it is tangential to the ROC curve. The point of tangency is the optimal operating point shown as the blue point in Fig. 6.1. This point presents the lowest expected cost with the given cost information. The threshold determined at this point is optimal with respect to the diagnostic performance and its corresponding expected cost. 


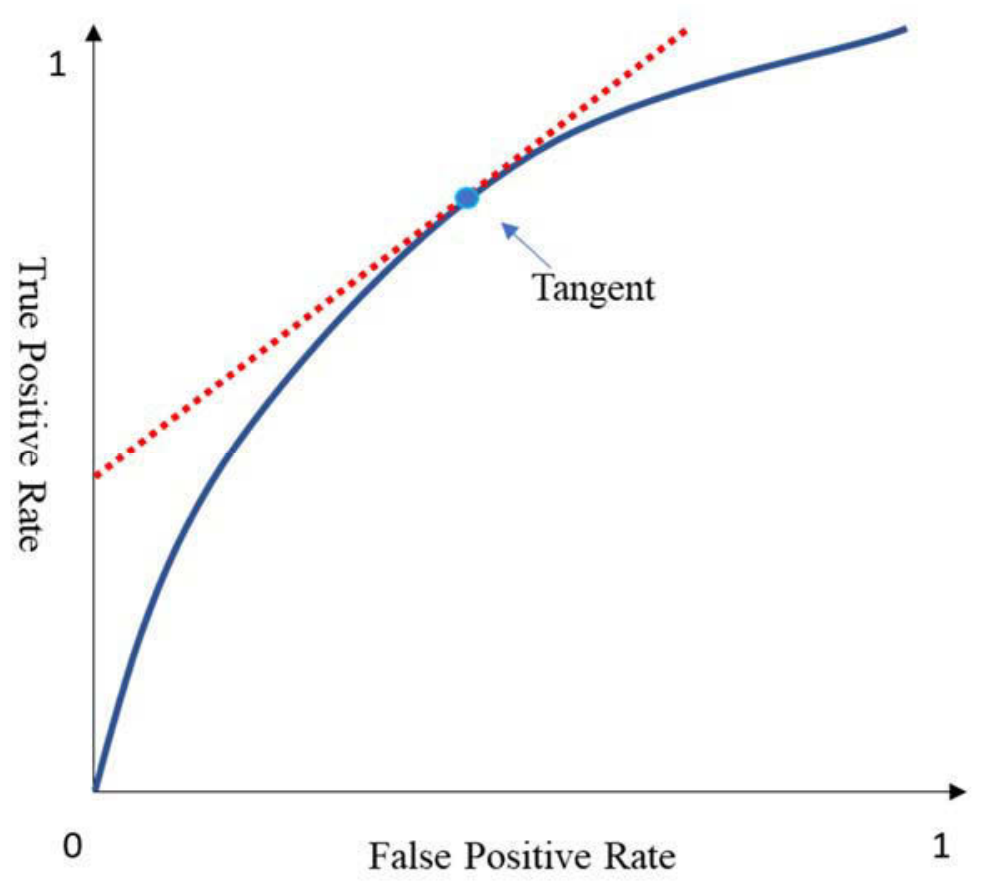

Figure 6.1 Optimal operating point within the ROC

\subsection{ROC Results and Analysis}

ROC curves of the three indicators are illustrated in Fig. 6.2. The left top corner, point $[0,1]$ implies the best performance in the ROC plot. That means all positive conditions or failures are detected and no false alarms occur. The closer the curve approaches to this point, the better it demonstrates diagnostic performance. From around 0.4 in TPR, the joint indicator inclines more to the left top corner at various TPR levels comparing to the other two individual indicators. That means the joint indicator detects more failures at the same false alarm ratio, showing superior diagnostic performance. For example, at $\mathrm{FPR}=0.04$, the joint indicator attains around 0.92 in TPR, whereas the two individual indicators barely reach 0.85 in TPR. 


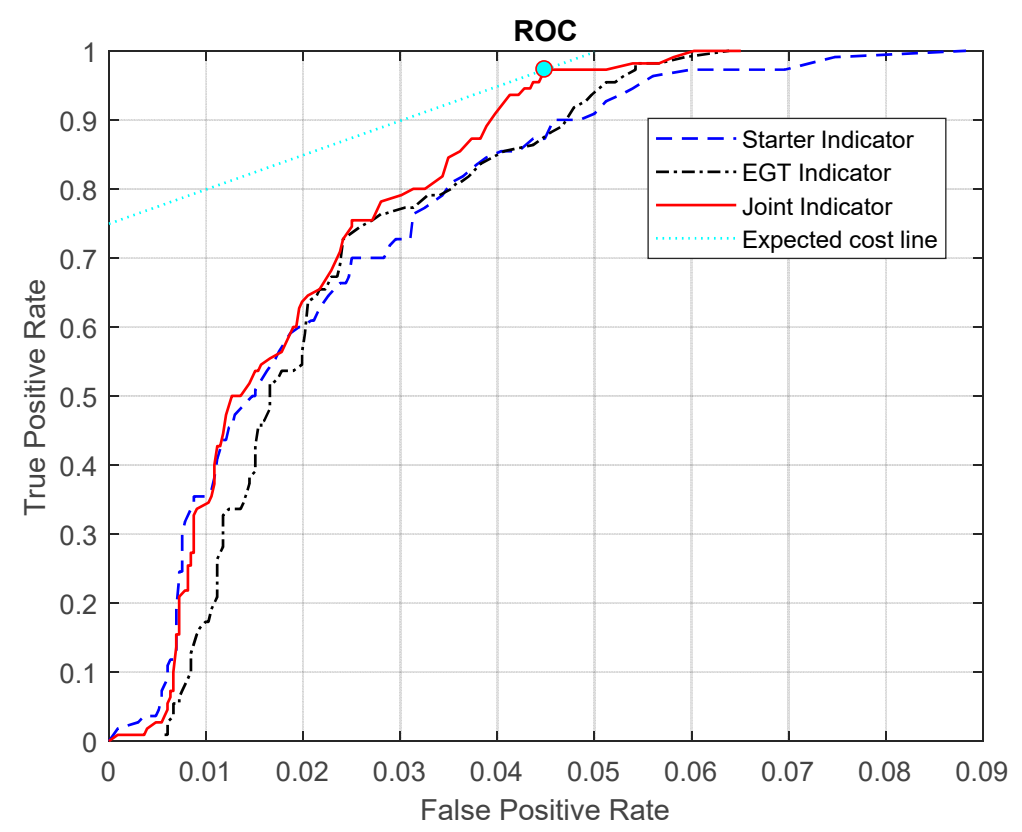

Figure 6.2 ROC curves of the indicators

\subsection{Expected Cost Results and Analysis}

Some costs such as built-in test and routine inspection incur regardless of the type of the indicators employed. Such costs are not included in the cost evaluation in this study. As expected, the correct detections do not lead to additional cost, therefore, $\operatorname{Cost}(P \mid P)=\$ 0$ and $\operatorname{Cost}(N \mid N)=\$ 0[91],[95]$.

When the health state of the starter is falsely detected as the faulty condition, the cost of installing a new starter is applied. Since the starter is a line replaceable unit (LRU), only a replacement operating is needed for the maintenance after the indication that will not delay the flight. The typical cost is $\operatorname{Cost}(P \mid N)=\$ 2580$, based on the purchase order list. When a faulty starter is detected as healthy, the starter failure can not be predicted, and a thorough inspection is required to identify the failure component. That leads to potential delays and even a cancellation of the flight. According to the report 'THE 
CONNECTED AIRCRAFT: Improving Dispatch Reliability', the average cost of a cancellation is $\operatorname{Cost}(N \mid P)=\$ 15650$ [96].

With above cost information, the slope of the straight line is obtained by Eq. (6.4) and the point of tangency between the straight line and ROC curves are determined, as shown in Fig. 6.2. The optimal operating point of the joint indicator is shown as the blue point in the plot. The optimal threshold under such cost consideration is also obtained, which incurs at 93\% fault detection probability.

Confusion matrices at this optimal threshold are provided in Table. 6.2. to compare the detection performance of the three indicators.

Table 6.2 Confusion matrices for the three indicators

\begin{tabular}{|c|c|c|c|c|c|c|c|}
\hline & \multicolumn{6}{|c|}{ True condition } \\
\hline & & \multicolumn{2}{|c|}{ The Joint Indicator } & \multicolumn{2}{|c|}{$\begin{array}{l}\text { Starter Health } \\
\text { Indicator }\end{array}$} & \multicolumn{2}{|c|}{$\begin{array}{c}E G T \text { Deviation } \\
\text { indicator }\end{array}$} \\
\hline & & Failure & Health & Failure & Health & Failure & Health \\
\hline \multirow{2}{*}{$\begin{array}{l}\text { Predicted } \\
\text { condition }\end{array}$} & Failure & 107 & 149 & 104 & 179 & 105 & 173 \\
\hline & Health & 3 & 3170 & 6 & 3140 & 5 & 3146 \\
\hline \multicolumn{2}{|c|}{$\begin{array}{c}\text { Diagnostic } \\
\text { performance }\end{array}$} & \multicolumn{2}{|c|}{$\begin{array}{c}\mathrm{TPR}=97.3 \% \\
\mathrm{FPR}=4.5 \% \\
\mathrm{ACC}=95.6 \%\end{array}$} & \multicolumn{2}{|c|}{$\begin{array}{c}\mathrm{TPR}=94.5 \% \\
\mathrm{FPR}=5.4 \% \\
\mathrm{ACC}=94.6 \%\end{array}$} & \multicolumn{2}{|c|}{$\begin{array}{c}\mathrm{TPR}=95.5 \% \\
\mathrm{FPR}=5.2 \% \\
\mathrm{ACC}=94.8 \%\end{array}$} \\
\hline \multicolumn{2}{|c|}{$\begin{array}{l}\text { Improvement } \\
\text { comparing to } \\
\text { individual indicators }\end{array}$} & & & \multicolumn{2}{|c|}{$\begin{array}{l}\text { TPR: } 3.0 \% \\
\text { FPR: }-17 \% \\
\text { ACC: } 1 \%\end{array}$} & \multicolumn{2}{|c|}{$\begin{array}{l}\text { TPR: } 1.9 \% \\
\text { FPR: }-13 \% \\
\text { ACC: } 0.8 \%\end{array}$} \\
\hline
\end{tabular}

The joint indicator misses 3 failures while 6 and 5 failures are missed by the starter health indicator and the EGT deviation indicator respectively. The joint indicator detects the most of failures, i.e., having the highest TPR, while maintaining the lowest FPR. The joint indicator detects $3.0 \%$ and $1.9 \%$ more faulty cases comparing to the starter health 
indicator and the EGT deviation indicator respectively, and FPR declines $17 \%$ and $13 \%$ compared to the mentioned indicators respectively. Its corresponding diagnostic accuracy (ACC) is the highest among the three indicators that outperforms the individual indicators by $1 \%$ and $0.8 \%$ respectively. It is obvious that the joint indicator exhibits superior diagnostic performance at this optimal threshold.

Based on the same cost consideration, expected costs of the three indicators at various threshold settings are calculated and the region above $90 \%$ fault detection probability is shown in Fig. 6.3, which is of most interest in practice.

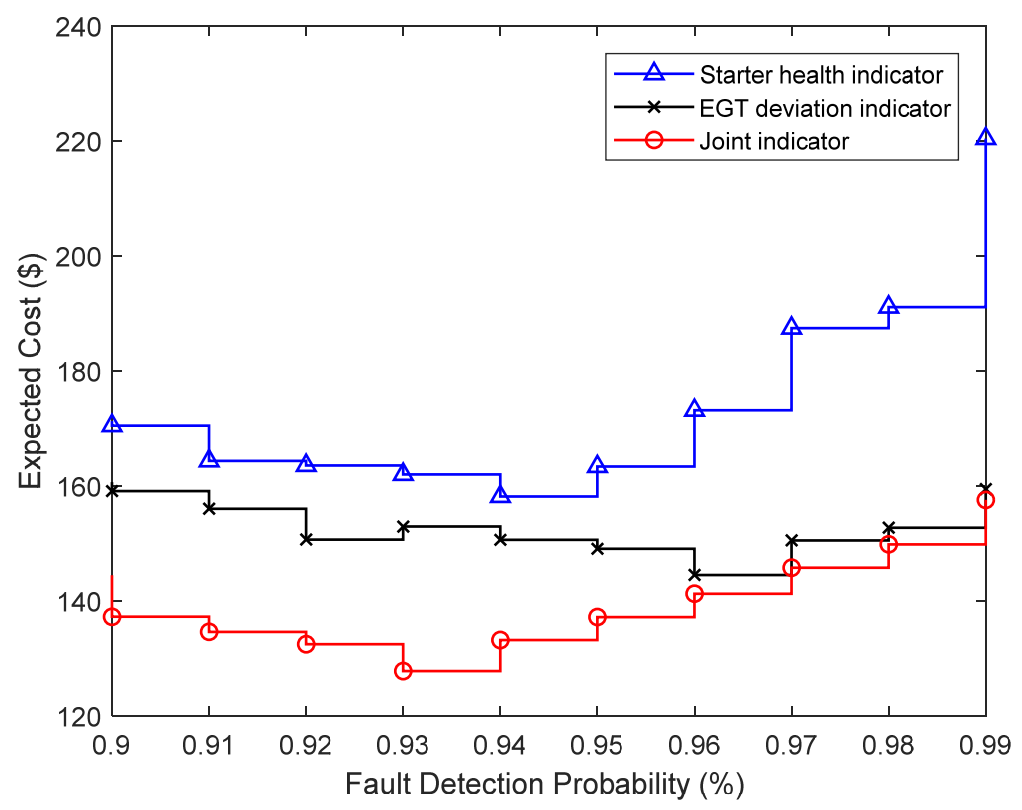

Figure 6.3 Expected costs at various fault detection probability

The joint indicator presents the lowest expected costs at its optimal threshold that incurs at $93 \%$ fault detection probability. The optimal thresholds for the starter health indicator and the EGT deviation indicator incur at $94 \%$ and $96 \%$ respectively. At their 
respective optimal thresholds, both indicators show the lowest expected cost during their entire detecting range. However, no matter what threshold is selected within the shown range, the joint indicator always exhibits the lowest expected cost among the three indicators.

For evaluating the cost of the three indicators, the numbers provided here are representative values extracted from "THE CONNECTED AIRCRAFT: Improving Dispatch Reliability" report [95]. In practice, the cost varies from application to application and from time to time. As a result more complex situations should be addressed. This requires extensive business information from the specific cases.

\subsection{Summary}

The diagnostic performance of the indicators is evaluated by using ROC and the optimal threshold is determined based on the trade-off between improving detection reliability and reducing operating costs. At the optimal threshold, the joint indicator detects $3.0 \%$ and $1.9 \%$ more faulty cases comparing to the starter health indicator and the EGT deviation indicator respectively, whereas FPR declines $17 \%$ and $13 \%$ contrasting with individual indicators. And the diagnostic accuracy increases 1\% and $0.8 \%$ separately. The joint indicator presents a predominant diagnostic capability at various thresholds and offers a significant improvement in overall maintenance costs. 


\section{Chapter: Conclusions and Future work}

\subsection{Contributions}

The key contributions of the developed diagnostic techniques to the field of the expert knowledge are:

1. The physics-based model for the APU startup phase does not require component characteristics and uses a generic approach to establish mathematical models for the compressor and the turbine;

2. The physics-based model presents a deep physical understanding of the gas turbine compared to the existing data-driven and statistic methods, while it provides guidance for the input parameter selection of the neural network methodology;

3. Both physics-based and neural network models show clear symptoms for the starter degradation, which is necessary for the predictive maintenance;

4. Two physics-based indicators possess tangible physical meanings and perform diagnosis in real-time, without a need to the historical data from the measurements;

5. The starter health indicator is developed specifically for the starter faults rather than other components of the APU, which is the first indicator defined at the component level;

6. The enhanced joint indicator defines a nonlinear threshold in the two-dimensional feature space, which enables multiple-feature diagnosis;

7. All required data are readily available from the existing sensors on the aircraft and no additional sensors are needed for the proposed diagnostic approach. 


\subsection{Summary and Conclusions}

An electric starter provides initial power to accelerate the APU during the startup phase. With the starter degradation, its output power declines, affecting the APU starting performance, and eventually resulting in a starting failure. This may lead to unexpected costs and increase the safety risk. Prior works mostly focus on presenting the degradation trend of the starter rather than quantitative health level of the system, which could not provide reliable information to support CBM. The theme of this thesis is to develop a physics-based diagnostic scheme to estimate the starter performance and diagnose its faults. In this study, a thermodynamic model associated with the transient operation of the APU during the starting period is established first. This model establishes the connection between APU measurement parameters and the starter performance. The performance estimation of the proposed physics-based model is compared with that of a neural-network-based data-driven model, which exhibits the strengths and weaknesses of both approaches. To quantitively monitor the health condition of the starter, two individual health indicators are derived from the physics-based model. Both indicators hold explicit physical meanings and are able to detect the failure in real-time without historical measurement data. To improve the diagnostic performance, an enhanced joint indicator is developed in the two-dimensional feature space that aggregates two indicators together to detect the failure simultaneously. ROC curves are introduced to evaluate the diagnostic performance of the three indicators and the optimal thresholds are determined based on the trade-off between the diagnostic reliability and the operating cost reduction. The enhanced joint indicator exhibits a superior diagnostic performance and offers a significant potential for overall maintenance costs reduction. The developed 
physics-based performance estimation and fault diagnostics scheme for the starter of APUs enables CBM for the starter and consequently warrants the safe operation of the starting system of APU.

\subsection{Recommendations for Future Work}

To enhance the achievement, there are some techniques to be involved in the future research.

- The proposed physics-based model associated with the transient operation of the APU during the starting period will be employed to other gas turbines for diagnostics and prognostics.

- This physics-based model established here can predict the performance parameters of the gas turbine. Any component of the APU can be monitored based on the same approach only if their deterioration is reflected by performance parameters.

- With characteristic maps and information of a gas turbine, the predicted performance from the physics-based model will improve the modeling accuracy.

- A data-model fusion approach shall be employed to improve the results.

- Accurate business information on specific cases in the study shall provide substantial information to explore optimal thresholds. 


\section{Bibliography or References}

[1] AIRBUS, “A310 Flight Crew Operating Manual,” 2008.

[2] W. O. L. Vianna, J. P. P. Gomes, R. K. H. Galvão, T. Yoneyama, and J. P. Matsuura, "Health Monitoring of an Auxiliary Power Unit Using a Classification Tree," in Proceedings of Annual Conference of the Prognostics and Health Management Society, 2011.

[3] A. K. S. Jardine, D. Lin, and D. Banjevic, "A review on machinery diagnostics and prognostics implementing condition-based maintenance," Mech. Syst. Signal Process., vol. 20, no. 7, pp. 1483-1510, Oct. 2006.

[4] Y. Zhao, V. Volovoi, M. Waters, and D. Mavris, "A Sequential Approach for Gas Turbine Power Plant Preventative Maintenance Scheduling," J. Eng. Gas Turbines Power, vol. 128, no. 4, pp. 796, Oct. 2006.

[5] R. Kothamasu, S. H. Huang, and W. H. VerDuin, "System health monitoring and prognostics - a review of current paradigms and practices," Int. J. Adv. Manuf. Technol., vol. 28, no. 9-10, pp. 1012-1024, Jul. 2006.

[6] H. Hanachi, C. Mechefske, J. Liu, A. Banerjee, and Y. Chen, "Performance-Based Gas Turbine Health Monitoring, Diagnostics, and Prognostics: A Survey," IEEE Trans. Reliab., pp. 1-24, 2018.

[7] E. L. Ntantis and P. N. Botsaris, "Diagnostic Methods for an Aircraft Engine Performance,” J. Eng. Sci. Technol. Rev., vol. 8, no. 4, pp. 1-10, 2015.

[8] Jie Liu, W. Wang, and F. Golnaraghi, "An Enhanced Diagnostic Scheme for Bearing Condition Monitoring," IEEE Trans. Instrum. Meas., vol. 59, no. 2, pp. 
309-321, Feb. 2010.

[9] Y. G. Li, "Performance-analysis-based gas turbine diagnostics: A review," Proc. Inst. Mech. Eng. Part A J. Power Energy, vol. 216, no. 5, pp. 363-377, Jan. 2002.

[10] R. Ganguli, Gas Turbine Diagnostics. CRC Press, 2012.

[11] C. Yang, M. Zaluski, and E. Scarlett, "APU FMEA validation and its application to fault identification," Proc. ASME 2010 Int. Des. Eng. Tech. Conf. Comput. Inf. Eng. Conf., 2010.

[12] K. Kim, O. Uluyol, G. Parthasarathy, and D. Mylaraswamy, "Fault Diagnosis of gas turbine engine LRUs Using the startup characteristics," Annu. Conf. Progn. Heal. Manag. Soc., vol. 3, 2012.

[13] M. L. Verbist, W. P. J. Visser, and J. P. van Buijtenen, "Experience With Gas Path Analysis for On-Wing Turbofan Condition Monitoring," J. Eng. Gas Turbines Power, vol. 136, no. 1, pp. 011204, Oct. 2013.

[14] P. Kamboukos and K. Mathioudakis, "Comparison of Linear and Non-Linear Gas Turbine Performance Diagnostics," in ASME Turbo Expo 2003, collocated with the 2003 International Joint Power Generation Conference. American Society of Mechanical Engineers, pp. 451-459, 2003.

[15] Y. G. Li, "Gas Turbine Performance and Health Status Estimation Using Adaptive Gas Path Analysis," J. Eng. Gas Turbines Power, vol. 132, no. 4, pp. 041701, Apr. 2010.

[16] Y. Liu and M. Su, "Nonlinear Model Based Diagnostic of Gas Turbine Faults: A Case Study," in ASME 2011 Turbo Expo: Turbine Technical Conference and Exposition. American Society of Mechanical Engineers, 2011, pp. 1-8. 
[17] L. A. Urban, "Parameter selection for multiple fault diagnostics of gas turbine engines," ASME J. Eng. Power, vol. 97, no. 2, pp. 225-230, 1975.

[18] L. Urban, Gas turbine engine parameter interrelationships. Hamilton Standard Division of United Aircraft Corporation, 1969.

[19] L. A. Urban, "Gas Path Analysis Applied to Turbine Engine Condition Monitoring," J. Aircr., vol. 10, no. 7, 1973.

[20] S. O. T. Ogaji, S. Sampath, R. Singh, and S. D. Probert, "Parameter selection for diagnosing a gas-turbine's performance-deterioration," Appl. Energy, vol. 73, no. 1, pp. 25-46, Sep. 2002.

[21] M. S. Grewal, "Gas turbine engine performance deterioration modelling and analysis," Cranfield Institute of Technology, 1988.

[22] H. I. H. Saravanamuttoo and F. S. Mirza-Baig, "Off-design Performance Prediction of Turbofans Using Gas Dynamics," Can. Aeronaut. Sp. J., vol. 39, no. 1, pp. 16-22, 1993.

[23] H. I. H. Saravanamuttoo, G. F. C. Rogers, and H. Cohen, Gas Turbine Theory. Pearson Education, 2001.

[24] H. I. H. Saravanamuttoo, "A Low Cost, On-Site Performance Monitoring System," in ASME 1979 International Gas Turbine Conference and Exhibit and Solar Energy Conference. American Society of Mechanical Engineers, pp. V01AT01A021-V01AT01A021, 1979.

[25] G. F. Aker and H. I. H. Saravanamuttoo, "Predicting Gas Turbine Performance Degradation Due to Compressor Fouling Using Computer Simulation Techniques," J. Eng. Gas Turbines Power, vol. 111, no. 2, pp. 343, Apr. 1989. 
[26] H. I. H. Saravanamuttoo, B. D. Maclsaac, L. Ottawa, and C. A. Member, "Thermodynamic models for pipeline gas turbine diagnostics," in ASME 1983 International Gas Turbine Conference and Exhibit. American Society of Mechanical Engineers, 1983.

[27] H. Hanachi, J. Liu, A. Banerjee, Y. Chen, and A. Koul, "A physics-based performance indicator for gas turbine engines under variable operating conditions," in ASME Turbo Expo 2014: Turbine Technical Conference and Exposition. American Society of Mechanical Engineers, 2014.

[28] F. S. Mibza-Bail' and H. I. H. Saravanamuttoot, "Off-Design Performance Prediction of Turbofans using Gasdynamics," in ASME 1991 International Gas Turbine and Aeroengine Congress and Exposition. American Society of Mechanical Engineers, 1991.

[29] J. K. Suraweera, "Off-design performance prediction of gas turbines without the use of compressor or turbine characteristics," Doctoral Dissertation, Carleton University, 2011.

[30] H. Wittenberg, "Prediction of Off-design Performance of Turbo-shaft Engines a Simplified Method," Seventh Eur. Rotorcr. powered lift Aircr. forum, no. 15, 1981.

[31] H. I. H. Saravanamuttoo and B. D. MacIsaac, "The Use of a Hybrid Computer in the Optimization of Gas Turbine Control Parameters," J. Eng. Power, vol. 95, no. 3, pp. 257, Jul. 1973.

[32] J. H. Kim, T. W. Song, T. S. Kim, and S. T. Ro, "Model Development and Simulation of Transient Behavior of Heavy Duty Gas Turbines," J. Eng. Gas Turbines Power, vol. 123, no. 3, pp. 589, Jul. 2001. 
[33] R. K. Agrawal and M. Yunis, "A Generalized Mathematical Model to Estimate Gas Turbine Starting,” J. Eng. Power, vol. 104, no. January, pp. 194-201, 1982.

[34] W. I. Rowen, "Simplified Mathematical Representations of Heavy-Duty Gas Turbines," J. Eng. Power, vol. 105, no. 83, pp. 865, 1983.

[35] M. R. Bank Tavakoli, B. Vahidi, and W. Gawlik, "An educational guide to extract the parameters of heavy duty gas turbines model in dynamic studies based on operational data," IEEE Trans. Power Syst., vol. 24, no. 3, pp. 1366-1374, 2009.

[36] A. Heng, S. Zhang, A. C. C. Tan, and J. Mathew, "Rotating machinery prognostics: State of the art, challenges and opportunities," Mech. Syst. Signal Process., vol. 23, no. 3, pp. 724-739, Apr. 2009.

[37] L. Marinai, D. Probert, and R. Singh, "Prospects for aero gas-turbine diagnostics: a review," Appl. Energy, vol. 79, no. 1, pp. 109-126, Sep. 2004.

[38] M. Zedda, "Gas turbine engine and sensor fault diagnosis," Doctoral Dissertation, Cranfield University, 1999.

[39] M. Zedda and R. Singh, "Gas Turbine Engine and Sensor Fault Diagnosis Using Optimization Techniques," J. Propuls. POWER, vol. 18, no. 5. pp. 1019-1025, 2002.

[40] A. M. Dietz W E, Kiech E L, "Jet and rocket engine fault diagnosis in real time," J. Neural Netw. Comput., vol. 1, no. 1, pp. 5-18, 1989.

[41] A. J. Volponi, H. DePold, R. Ganguli, and C. Daguang, "The Use of Kalman Filter and Neural Network Methodologies in Gas Turbine Performance Diagnostics: A Comparative Study," J. Eng. Gas Turbines Power, vol. 125, no. 4, pp. 917, Oct. 2003. 
[42] H. R. DePold and F. D. Gass, "The Application of Expert Systems and Neural Networks to Gas Turbine Prognostics and Diagnostics," in ASME 1998 International Gas Turbine and Aeroengine Congress and Exhibition. American Society of Mechanical Engineers, pp. V005T15A009, 1998.

[43] S. Sina Tayarani-Bathaie, Z. N. Sadough Vanini, and K. Khorasani, "Dynamic neural network-based fault diagnosis of gas turbine engines," Neurocomputing, vol. 125, pp. 153-165, Feb. 2014.

[44] R. B. Joly, S. O. T. Ogaji, R. Singh, and S. D. Probert, "Gas-turbine diagnostics using artificial neural-networks for a high bypass ratio military turbofan engine," Appl. Energy, vol. 78, no. 4, pp. 397-418, Aug. 2004.

[45] T. J. Ross, Fuzzy Logic with Engineering Applications. Chichester, UK: John Wiley \& Sons, Ltd, 2010.

[46] B. Kosko, Fuzzy engineering. Prentice Hall, 1997.

[47] R. Ganguli, “Application of Fuzzy Logic for Fault Isolation of Jet Engines," in ASME Turbo Expo 2001: Power for Land, Sea, and Air. American Society of Mechanical Engineers, pp. V004T04A006, 2001.

[48] S. O. T. Ogaji, L. Marinai, S. Sampath, R. Singh, and S. D. Prober, "Gas-turbine fault diagnostics: a fuzzy-logic approach," Appl. Energy, vol. 82, no. 1, pp. 81-89, Sep. 2005.

[49] R. Ganguli, "Fuzzy Logic Intelligent System for Gas Turbine Module and System Fault Isolation,” J. Propuls. Power, vol. 18, no. 2, pp. 440-447, Mar. 2002.

[50] R. W. Eustace, "A Real-World Application of Fuzzy Logic and Influence Coefficients for Gas Turbine Performance Diagnostics," J. Eng. Gas Turbines 
Power, vol. 130, no. 6, pp. 061601, Nov. 2008.

[51] J. S. Jang, "ANFIS: adaptive-network-based fuzzy inference system," IEEE Trans. Syst. Man. Cybern., vol. 23, no. 3, pp. 665-685, 1993.

[52] K. Salahshoor, M. Kordestani, and M. S. Khoshro, "Fault detection and diagnosis of an industrial steam turbine using fusion of SVM (support vector machine) and ANFIS (adaptive neuro-fuzzy inference system) classifiers," Energy, vol. 35, no. 12, pp. 5472-5482, Dec. 2010.

[53] I. Steinwart and A. Christmann, Support vector machines. Springer, 2008.

[54] C. Cortes and V. Vapnik, "Support-vector networks," Mach. Learn., vol. 20, no. 3, pp. 273-297, Sep. 1995.

[55] D. Zhou, H. Zhang, and S. Weng, "A New Gas Path Fault Diagnostic Method of Gas Turbine Based on Support Vector Machine," J. Eng. Gas Turbines Power, vol. 137, no. 10, pp. 102605, Oct. 2015.

[56] P. Walsh and P. Fletcher, Gas turbine performance. John Wiley \& Sons, 2004.

[57] K. Kim and D. Mylaraswamy, "Fault Diagnosis and Prognosis of Gas Turbine Engines Based on Qualitative Modeling," in ASME Turbo Expo 2006: Power for Land, Sea, and Air. American Society of Mechanical Engineers, pp. 881-889, 2006.

[58] Q. Lou, "Aircraft APU Starter Health Monitoring and Failure Prognostics," M. S. thesis, Carleton University, 2013.

[59] C. Yang, Q. Lou, J. Liu, Y. Yang, and Q. Cheng, "Particle filtering-based methods for time to failure estimation with a real-world prognostic application," Appl. Intell., vol. 48, no. 8, pp. 2516-2526, Aug. 2018. 
[60] J. M. Peña, S. Létourneau, and F. Famili, "Application of Rough Sets Algorithms to Prediction of Aircraft Component Failure," in Proceedings of the Third International Symposium on Intelligent Data Analysis, pp. 473-484, 1999.

[61] L. M. Stohlgren, “The GTCP331, A 600 hp Auxiliary Power Unit Program,” in ASME 1983 International Gas Turbine Conference and Exhibit. American Society of Mechanical Engineers, pp. V002T02A013-V002T02A013, 1983.

[62] D. Gorinevsky, K. Dittmar, H. E. Systems, D. Mylaraswamy, and E. Nwadiogbu, "Model-based Diagnostics for an Aircraft Auxiliary Power Unit," in Proceedings of the 41st IEEE Conference on Decision and Control, no. 208, pp. 1-6, 2002.

[63] S. Letourneau, C. Yang, and Z. Liu, "Improving Preciseness of Time to Failure Predictions: Application to APU starter," in 2008 International Conference on Prognostics and Health Management, pp. 1-7, 2008.

[64] C. Yang, Q. Lou, J. Liu, H. Gou, and Y. Bai, "Particle Filter-Based Approach to Estimate Remaining Useful Life for Predictive Maintenance,” in International Conference on Industrial, Engineering and Other Applications of Applied Intelligent Systems, pp. 692-701, 2015.

[65] C. Yang, S. Letourneau, J. Liu, Q. Cheng, and Y. Yang, "Machine Learning-based Methods for TTF Estimation with Application to APU Prognostics," Appl. Intell., vol. 46, no. 1, pp. 227-239, Jan. 2017.

[66] S. R. Gaudet and J. E. D. Gauthier, "A Simple Sub-idle Component Map Extrapolation Method," ASME Turbo Expo 2007 Power Land, Sea, Air. Am. Soc. Mech. Eng., pp. 29-37, 2007.

[67] M. Pakmehr, N. Fitzgerald, E. Feron, J. Paduano, and A. Behbahani, "Physics- 
Based Dynamic Modeling of a Turboshaft Engine Driving a Variable Pitch Propeller," J. Propuls. Power, vol. 32, no. 3, 2016.

[68] C. Rodgers, "Typical Performance Characteristics of Gas Turbine," J. Eng. Power, vol. 86, no. 2, pp. 161-170, 1964.

[69] M. Casey and C. Robinson, "A Method to Estimate the Performance Map of a Centrifugal Compressor Stage,” J. Turbomach., vol. 135, no. 2, pp. 021-034, 2012.

[70] N. A. Cumpsty, Compressor aerodynamics. Longman Scientific \& Technical, 1989.

[71] M. V. Casey, "The Effects of Reynolds Number on the Efficiency of Centrifugal Compressor Stages,” J. Eng. Gas Turbines Power, vol. 107, no. 2, pp. 541-548, 1985.

[72] D. H. Mallinson and W. G. E. Lewis, "The part-load performance of various gasturbine engine schemes," Proc. Inst. Mech. Eng., vol. 159, no. 1, pp. 198-219, 1948.

[73] D. H. Cooke, "On Prediction of Off-Design Multistage Turbine Pressures by Stodola's Ellipse," J. Eng. Gas Turbines Power, vol. 107, no. 3, pp. 596, Jul. 1985.

[74] B. MacIsaac and R. Langton, Gas Turbine Propulsion Systems. 2011.

[75] D. Gorinevsky, E. Nwadiogbu, and D. Mylaraswamy, "Model-based Diagnostics for Small-scale Turbomachines," Proc. 41st IEEE Conf. Decis. Control. 2002., vol. 4, pp. 4784-4789, 2002.

[76] H. Hanachi, J. Liu, A. Banerjee, Y. Chen, and A. Koul, "A Physics-Based Modeling Approach for Performance Monitoring in Gas Turbine Engines," IEEE 
Trans. Reliab., vol. 64, no. 1, pp. 197-205, 2015.

[77] A. Lazzaretto and A. Toffolo, "Analytical and Neural Network Models for Gas Turbine Design and Off-design Simulation," Int. J. Thermodyn., vol. 4, no. 4, pp. 173-182, 2001.

[78] K. Kanelopoulos and A. Stamatis, "Incorporating Neural Networks into Gas Turbine Performance Diagnostics," in ASME 1997 International Gas Turbine and Aeroengine Congress and Exhibition, pp. V004T15A011-V004T15A011, 1997.

[79] S. O. T. Ogaji, Y. G. Li, S. Sampath, and R. Singh, "Gas Path Fault Diagnosis of a Turbofan Engine From Transient Data Using Artificial Neural Networks,” in ASME Turbo Expo 2003, collocated with the 2003 International Joint Power Generation Conference. American Society of Mechanical Engineers, pp. 405-414, 2003.

[80] P.J. Lu, M.-C. Zhang, T.-C. Hsu, and J. Zhang, “An Evaluation of Engine Faults Diagnostics Using Artificial Neural Networks," in ASME Turbo Expo 2000: Power for Land, Sea, and Air. American Society of Mechanical Engineers, pp. V004T04A001, 2000.

[81] H. Demuth and B. De Jesús, Neural Network Design. Martin Hagan, 2014.

[82] S. S. Haykin, Neural Networks: a Comprehensive Foundation. Prentice Hall, 1999.

[83] K. Hornik, M. Stinchcombe, and H. White, "Multilayer Feedforward Networks are Universal Approximators," Neural Networks, vol. 2, no. 5, pp. 359-366, Jan. 1989.

[84] M. H. Beale, M. T. Hagan, and H. B. Demuth, "Neural Network Toolbox ${ }^{\text {TM }}$ User's Guide,” R2017a, The MathWorks, Inc., 2017. 
[85] Q. Zhou, J. Son, S. Zhou, X. Mao, and M. Salman, "Remaining Useful Life Prediction of Individual Units Subject to Hard Failure," IIE Trans., vol. 46, no. 10, pp. 1017-1030, Oct. 2014.

[86] X. Flandrois, J. Lacaille, J.-R. Masse, and A. Ausloos, "Expertise Transfer and Automatic Failure Classification for the Engine Start Capability System," in AIAA Infotech@Aerospace Conference, 2009.

[87] A. Stamatis, K. Mathioudakis, M. Smith, and K. Papailiou, "Gas Turbine Component Fault Identification by Means of Adaptive Performance Modeling," in ASME 1990 International Gas Turbine and Aeroengine Congress and Exposition. American Society of Mechanical Engineers, pp. V005T15A015, 1990.

[88] M. P. Wand and M. C. Jones, Kernel Smoothing. Chapman \& Hall, 1995.

[89] B. W. Silverman, Density estimation for statistics and data analysis. Chapman and Hall, 1986.

[90] T. Fawcett, "ROC graphs: Notes and practical considerations for researchers," Mach. Learn., vol. 31, no. 1, pp. 1-38, 2004.

[91] N. M. Adams and D. J. Hand, "Comparing classifiers when the misallocation costs are uncertain," Pattern Recognit., vol. 32, no. 7, pp. 1139-1147, Jul. 1999.

[92] P. Pillai, A. Kaushik, S. Bhavikatti, A. Roy, and V. Kumar, "A Hybrid Approach for Fusing Physics and Data for Failure Prediction," Int. J. Progn. Heal. Manag., vol. 7, no. 025 , pp. 1-12, 2016.

[93] J. Swets, "The science of choosing the right decision threshold in high-stakes diagnostics.," Am. Psychol., vol. 47, no. 2, pp. 522, 1992.

[94] T. Fawcett, "An introduction to ROC analysis," Pattern Recognit. Lett., vol. 27, 
no. 8, pp. 861-874, Jun. 2006.

[95] R. Bettinger, "Cost-Sensitive Classifier Selection Using the ROC Convex Hull Method," SAS Inst., pp. 1-12, 2003.

[96] T. Larsen, "THE CONNECTED AIRCRAFT: Improving Dispatch Reliability," in World Maintenance Symposium, pp. 25, 2005. 